\title{
A Family of Optimal Eighth Order Iteration Functions for Multiple Roots and Its Dynamics
}

\author{
Saima Akram (D), ${ }^{1}$ Faiza Akram $\left(D,{ }^{1}\right.$ Moin-ud-Din Junjua $\left(D,{ }^{2}\right.$ Misbah Arshad $\left(D,{ }^{3}\right.$ \\ and Tariq Afzal (iD) ${ }^{2}$ \\ ${ }^{1}$ Centre for Advanced Studies in Pure \& Applied Mathematics, Bahauddin Zakariya University Multan, Multan 60000, Pakistan \\ ${ }^{2}$ Department of Mathematics and Statistics, Institute of Southern Punjab Multan, Multan 60000, Pakistan \\ ${ }^{3}$ Department of Mathematics, COMSATS University Islamabad, Sahiwal Campus, Punjab 57000, Pakistan
}

Correspondence should be addressed to Saima Akram; saimaakram@bzu.edu.pk

Received 29 January 2021; Revised 17 February 2021; Accepted 27 February 2021; Published 15 March 2021

Academic Editor: Ghulam Mustafa

Copyright (C) 2021 Saima Akram et al. This is an open access article distributed under the Creative Commons Attribution License, which permits unrestricted use, distribution, and reproduction in any medium, provided the original work is properly cited.

In this manuscript, we present a new general family of optimal iterative methods for finding multiple roots of nonlinear equations with known multiplicity using weight functions. An extensive convergence analysis is presented to verify the optimal eighth order convergence of the new family. Some special cases of the family are also presented which require only three functions and one derivative evaluation at each iteration to reach optimal eighth order convergence. A variety of numerical test functions along with some real-world problems such as beam designing model and Van der Waals' equation of state are presented to ensure that the newly developed family efficiently competes with the other existing methods. The dynamical analysis of the proposed methods is also presented to validate the theoretical results by using graphical tools, termed as the basins of attraction.

\section{Introduction}

The solution of a nonlinear equation of the type, $g(x)=0$, is one of the important problems and is a demanding task in computational mathematics. This problem becomes more complex when we deal with multiple roots of nonlinear equations. Many problems arise in the areas of engineering and mathematical modeling with the need to determine roots of the nonlinear equations as an intermediate problem for their solution. We have a large number of one-point and multipoint methods in the literature which are used to find roots of nonlinear equations, see [1-4]. A natural question arises that, is there any need of these variants? This question is pinching and alarming. So, we decided to investigate for its answer. We have tested iterative schemes having the same order of convergence for different test functions. We have also chosen test functions from real-world problems such as Van der Waals' equation of state and beam positioning problem. It is observed that every variant behaves differently for different test functions, so the need for more and more variants is justified. It is almost fictitious to find solutions of such problems in analytical form. In practice, to obtain approximated and effective solution up to a specific degree of accuracy, we use an iterative procedure. In the past years, many researchers have been worked to formulate iterative multiple root finding schemes with higher order of convergence, by knowing the practical, challenging, and demanding nature of multiple zeros. The behavior of iterative schemes for multiple roots is not similar to that of simple ones of nonlinear equations.

The well-known classical Newton's method with quadratic order of convergence is given as [5]

$$
x_{\zeta+1}=x_{\zeta}-\frac{g\left(x_{\zeta}\right)}{g^{\prime}\left(x_{\zeta}\right)} .
$$

In case of multiple roots of nonlinear equations, this classical Newton's method fails to keep the quadratic convergence and drops to linear convergence, when provided a good initial guess $x_{0}$ near the exact root $\alpha$. Classical Newton's method with a prior knowledge of multiplicity $(k>1)$ yields a modified Newton's method also known as Rall's 
method [6] which converges quadratically to the desired multiple root, given as

$$
x_{\zeta+1}=x_{\zeta}-k \cdot \frac{g\left(x_{\zeta}\right)}{g^{\prime}\left(x_{\zeta}\right)}, \quad \zeta \geq 0 .
$$

In recent years, many researchers have presented optimal fourth order convergent schemes for multiple roots when multiplicity is known in advance [7-11]. Thukral [12], Geum et al. [13, 14], and Sharma et al. [15] presented nonoptimal sixth and seventh order multiple root finding methods. The optimal convergence order is defined by Kung and Traub [16] that a without memory method can accomplish the order of convergence at most $2^{n-1}$ consuming $n$ function or derivative evaluations. Efficiency index is defined by Ostrowski [17] as, if the order of convergence of an iterative family is $r$ and the total number of function or derivative evaluations per iteration is $n$, then the efficiency index of an iterative scheme is $r^{(1 / n)}$.

In 2018, Behl et al. [18] presented a class of optimal eighth order methods for approximating multiple roots of nonlinear equations with known multiplicity $k$, given as follows:

$$
\begin{gathered}
y_{\zeta}=x_{\zeta}-k \cdot \frac{g\left(x_{\zeta}\right)}{g^{\prime}\left(x_{\zeta}\right)}, \quad \zeta \geq 0, \\
z_{\zeta}=y_{\zeta}-G\left(t_{\zeta}\right) \cdot s_{\zeta} \cdot \frac{g\left(x_{\zeta}\right)}{g^{\prime}\left(x_{\zeta}\right)}, \\
x_{\zeta+1}=z_{\zeta}-H\left(s_{\zeta}, w_{\zeta}\right) \cdot s_{\zeta} \cdot w_{\zeta} \cdot \frac{g\left(x_{\zeta}\right)}{g^{\prime}\left(x_{\zeta}\right)},
\end{gathered}
$$

where $s_{\zeta}=\left[\left(g\left(y_{\zeta}\right) / g\left(x_{\zeta}\right)\right)\right]^{(1 / k)}, t_{\zeta}=\left(s_{\zeta} / \beta_{1}+\beta_{2} s_{\zeta}\right), w_{\zeta}$ $=\left[\left(g\left(z_{\zeta}\right) / g\left(y_{\zeta}\right)\right)\right]^{(1 / k)}, \beta_{1}$ and $\beta_{2}$ are parameters. The univariate weight function $G: \mathbb{C} \longrightarrow \mathbb{C}$ and bivariate weight function $H: \mathbb{C}^{2} \longrightarrow \mathbb{C}$ are analytic in a neighborhood of $(0)$ and $(0,0)$, respectively.

In 2019, Akram et al. [19] proposed an optimal eighth order multiple root finding scheme based on weight function approach with known multiplicity $(k \geq 1)$, which is given as follows:

$$
\begin{gathered}
y_{\zeta}=x_{\zeta}-k \cdot \frac{g\left(x_{\zeta}\right)}{g^{\prime}\left(x_{\zeta}\right)}, \quad \zeta \geq 0, \\
z_{\zeta}=y_{\zeta}-k \cdot G\left(s_{\zeta}\right) \cdot s_{\zeta} \cdot \frac{g\left(x_{\zeta}\right)}{g^{\prime}\left(x_{\zeta}\right)}, \\
x_{\zeta+1}=z_{\zeta}-k \cdot H\left(t_{\zeta}, w_{\zeta}\right) \cdot s_{\zeta} \cdot \frac{g\left(x_{\zeta}\right)}{g^{\prime}\left(x_{\zeta}\right)},
\end{gathered}
$$

where $s_{\zeta}=\left[\left(g\left(y_{\zeta}\right) / g\left(x_{\zeta}\right)\right)\right]^{(1 / k)}, t_{\zeta}=\left[\left(g\left(z_{\zeta}\right) / g\left(y_{\zeta}\right)\right)\right]^{(1}$ $/ k), w_{\zeta}=\left[\left(g\left(z_{\zeta}\right) / g\left(x_{\zeta}\right)\right)\right]^{(1 / k)}$. The univariate weight function $G: \mathbb{C} \longrightarrow \mathbb{C}$ is analytic and bivariate weight function
$H: \mathbb{C}^{2} \longrightarrow \mathbb{C}$ is holomorphic in a neighborhood of $(0)$ and $(0,0)$, respectively.

Zafar et al. [20] presented another family of optimal eighth order multiple root finders with known multiplicity by using free parameters $\lambda_{1}, \lambda_{2} \in \mathbb{R}$, given as follows:

$$
\begin{aligned}
& y_{\zeta}=x_{\zeta}-k \cdot \frac{g\left(x_{\zeta}\right)}{g^{\prime}\left(x_{\zeta}\right)}, \quad \zeta \geq 0, \\
& z_{\zeta}=y_{\zeta}-k \cdot G\left(s_{\zeta}\right) \cdot s_{\zeta} \cdot \frac{g\left(x_{\zeta}\right)}{g^{\prime}\left(x_{\zeta}\right)}, \\
& x_{\zeta+1}=z_{\zeta}-k \cdot H\left(t_{\zeta}\right) \cdot P\left(w_{\zeta}\right) \cdot\left(\lambda_{1}+\lambda_{2} s_{\zeta}\right) \cdot s_{\zeta} \cdot w_{\zeta} \cdot \frac{g\left(x_{\zeta}\right)}{g^{\prime}\left(x_{\zeta}\right)},
\end{aligned}
$$

where $s_{\zeta}=\left[\left(g\left(y_{\zeta}\right) / g\left(x_{\zeta}\right)\right)\right]^{(1 / k)}, w_{\zeta}=\left[\left(g\left(z_{\zeta}\right) / g\left(y_{\zeta}\right)\right)\right]^{(1}$ $/ k), t_{\zeta}=\left[\left(g\left(z_{\zeta}\right) / g\left(x_{\zeta}\right)\right)\right]^{(1 / k)}$. The univariate weight functions $G: \mathbb{C} \longrightarrow \mathbb{C}, H: \mathbb{C} \longrightarrow \mathbb{C}$, and $P: \mathbb{C} \longrightarrow \mathbb{C}$ are analytic in a neighborhood of (0). The above-presented schemes (3), (4), and (5) require only four-function or derivative evaluations and their efficiency index is $8^{(1 / 4)}=1.6818$.

There is very limited literature about the higher order optimal multiple root finding iterative schemes that can handle multiple roots with known multiplicity $(k \geq 1)$. The main reason is that it is a more challenging and time taking task with tough and lengthy computations to develop iterative methods for finding multiple roots. In the literature, most of the iterative procedures for multiple roots are the extensions of modified Newton's method with complex body structures.

Our aim for the presented work is to develop a general family of multiple root finding methods with simple and compact body structures. Therefore, with the demand to construct simple and more effective optimal higher order methods for multiple roots, we present a family of optimal eighth order convergent iterative methods. The proposed scheme requires only four-function evaluations per iterative step which satisfies the classical conjecture given by Kung and Traub [16] and thus falls in the category of optimal methods. The new simple structured scheme is based on univariate and trivariate weight functions in each iterative step. Our scheme provides faster convergence and wider regions of convergence as compared to the results obtained by the earlier methods of similar kind.

The rest of the manuscript is organized as follows: in Section 2, we present the development of the new family based on weight function approach for finding multiple roots with known multiplicity $(k \geq 1)$, followed by the analysis of its convergence to achieve the optimal eighth order of convergence. In Section 3, some simple special cases of weight functions and proposed family are presented. These special cases are used to perform the numerical tests and comparison of the performance of newly developed methods with the existing schemes is presented in Section 4. In Section 5, an extensive dynamical analysis of the presented methods in complex plane is shown by using a 
graphical tool, namely, basins of attraction. Finally, the concluding remarks are given in Section 6.

\section{Methodology of Iterative Scheme}

In this section, we define a general and simple iterative family in order to approximate multiple roots of nonlinear equations. Let $\alpha$ be a multiple root with known multiplicity $(k \geq 1)$ of the function $g: \mathbb{C} \longrightarrow \mathbb{C}$. We consider the following iterative scheme by employing weight functions at each iterative step:

$$
\begin{aligned}
y_{\zeta} & =x_{\zeta}-G\left(v_{\zeta}\right), \quad \zeta \geq 0, \\
z_{\zeta} & =y_{\zeta}-H\left(t_{\zeta}\right) \cdot G\left(v_{\zeta}\right), \\
x_{\zeta+1} & =z_{\zeta}-H\left(t_{\zeta}\right)\left(v_{\zeta}\right) \cdot D\left(t_{\zeta}, s_{\zeta}, w_{\zeta}\right) G,
\end{aligned}
$$

where $\quad v_{\zeta}=\left(g\left(x_{\zeta}\right) / g^{\prime}\left(x_{\zeta}\right)\right), t_{\zeta}=\left[\left(g\left(y_{\zeta}\right) / g\left(x_{\zeta}\right)\right)\right]^{(1 / k)}$, $s_{\zeta}=\left[\left(g\left(z_{\zeta}\right) / g\left(y_{\zeta}\right)\right)\right]^{(1 / k)}, w_{\zeta}=\left[\left(g\left(z_{\zeta}\right) / g\left(x_{\zeta}\right)\right)\right]^{(1 / k)}$.

The weight functions $G, H$, and $D$ involved in the above iterative scheme (6) play an eminent role to obtain optimal eighth order convergence. The univariate weight functions
$G: \mathbb{C} \longrightarrow \mathbb{C}$ and $H: \mathbb{C} \longrightarrow \mathbb{C}$ are analytic in the neighborhood of (0) and the trivariate weight function $D: \mathbb{C}^{3} \longrightarrow \mathbb{C}$ is analytic in the neighborhood of $(0,0,0)$. Note that the schemes given by (3), (4), and (5) are the special cases of our proposed family (6).

In the next theorem, we find the conditions on univariate and trivariate weight functions and investigate the optimal eighth order of convergence of presented scheme (6).

Theorem 1. Let $\alpha$ be a multiple root with multiplicity $(k \geq 1)$ of the involved function $g$ and $g: \mathbb{C} \longrightarrow \mathbb{C}$ is analytic in the region enclosing a multiple zero $\alpha$ of $g$. Further, we also suppose that $D: \mathbb{C}^{3} \longrightarrow \mathbb{C}, G: \mathbb{C} \longrightarrow \mathbb{C}$, and $H: \mathbb{C} \longrightarrow \mathbb{C}$ are analytic in neighborhood of their respected origins. Then, for an initial guess $x_{0}$ provided sufficiently close to $\alpha$ of $g$, the iterative method defined by (6) has an optimal eighth order of convergence when the following conditions hold: $A_{0}=0$; $A_{1}=k ; A_{2}=0 ; B_{0}=0 ; B_{1}=1 ; B_{2}=4 ; B_{3}=18 ; D_{000}=0$; $D_{100}=0 ; D_{010}=1 ; D_{200}=0 ; D_{001}=-1 ; D_{110}=1 ; D_{020}=2$; $D_{101}=1 ; D_{011}=2$, with the following error equation:

$$
e_{\zeta+1}=\frac{1}{24 k^{7}}\left\{C_{1}\left((3+k) C_{1}^{2}-2 k C_{2}\right)\left(\left(-163+7 k^{2}\right) C_{1}^{4}-24 k^{2} C_{1}^{2} C_{2}-12 k^{2} C_{2}^{2}+12 k^{2} C_{1} C_{3}\right) e_{\zeta}^{8}\right\}+O\left(e_{\zeta}^{9}\right)
$$

where $e_{\zeta}=x_{\zeta}-\alpha$ and $C_{t}=(k ! /(k+t) !)\left(g^{(k+t)}(\alpha) / g^{(k)}(\alpha)\right)$, for $t \in \mathbb{N}$.
Proof. Let $\alpha$ be the multiple zero of $g$ and $e_{\zeta}=x_{\zeta}-\alpha$ be the error at $\zeta^{\text {th }}$ iteration, where $k$ is the known multiplicity and $k \geq 1$. Now, we adopt the Taylor series expansion of $g\left(x_{\zeta}\right)$ and $g^{\prime}\left(x_{\zeta}\right)$ around $\alpha$; we have

$$
\begin{aligned}
& g\left(x_{\zeta}\right)=\frac{g^{(k)}(\alpha)}{k !} e_{\zeta}^{k}\left(1+C_{1} e_{\zeta}+C_{2} e_{\zeta}^{2}+C_{3} e_{\zeta}^{3}+C_{4} e_{\zeta}^{4}+C_{5} e_{\zeta}^{5}+C_{6} e_{\zeta}^{6}+C_{7} e_{\zeta}^{7}+C_{8} e_{\zeta}^{8}+O\left(e_{\zeta}^{9}\right)\right), \\
& g^{\prime}\left(x_{\zeta}\right)=\frac{g^{(k)}(\alpha)}{k !} e_{\zeta}^{k-1}\left(k+(k+1) C_{1} e_{\zeta}+(k+2) C_{2} e_{\zeta}^{2}+(k+3) C_{3} e_{\zeta}^{3}+(k+4) C_{4} e_{\zeta}^{4}+\cdots+O\left(e_{\zeta}^{9}\right)\right),
\end{aligned}
$$

respectively.

By using expressions (8) and (9), we get

$$
v_{\zeta}=\frac{1}{k} e_{\zeta}-\frac{C_{1}}{k} e_{\zeta}^{2}+\frac{(1+k)-2 k C_{1}}{k^{3}} e_{\zeta}^{3}+O\left(e_{\zeta}^{4}\right)
$$

We expand the weight function $G\left(v_{\zeta}\right)$ by using Taylor series, which leads us to the following expression:

$$
G\left(v_{\zeta}\right)=A_{0}+A_{1} v_{\zeta}+\frac{1}{2} A_{2} v_{\zeta}^{2}
$$

where $A_{j}=\left(A^{j}(0) / j !\right)$ for $0 \leq j \leq 2$.

By employing expression (11) in the first step of scheme (6), we have

$$
y_{\zeta}=-A_{0}+\left(1-\frac{A_{1}}{k}\right) e_{\zeta}-\left(\frac{-2 C_{1} A_{1}+A_{2}}{2 k^{2}}\right) e_{\zeta}^{2}+O\left(e_{\zeta}^{3}\right)
$$

To achieve the second order of convergence, we select $A_{0}=0$ and $A_{1}=k$ and obtain

$$
y_{\zeta}=\left(\frac{2 k C_{1}-A_{2}}{2 k^{2}}\right) e_{\zeta}^{2}+O\left(e_{\zeta}^{3}\right)
$$

By using expression (13) and Taylor series expansion of $y_{\zeta}$, we get the following expression: 


$$
\begin{aligned}
g\left(y_{\zeta}\right)= & g^{(k)}(\alpha) e^{2 k}\left(\frac{2^{-k}\left(-\left(A_{2}-2 k C_{1} / k^{2}\right)\right)^{k}}{k !}-\frac{2^{1-k}\left(A_{2}-2 k C_{1} / k^{2}\right)^{k-1}\left(-A_{2} C_{1}+k(1+k) C_{1}^{2}-2 k^{2} C_{2}\right)}{k^{2} k !}\right) e_{\zeta} \\
& +g^{(k)}(\alpha) e^{2 k}\left(\sum_{x=0}^{2} F_{x} e_{\zeta}^{x+2}\right)+O\left(e_{\zeta}^{5}\right),
\end{aligned}
$$

where $F_{x}=F_{x}\left(k, C_{1}, C_{2}, \ldots, C_{8}\right)$ are given in terms of $k, C_{1}, C_{2}, \ldots, C_{8}$. For example, the expressions for coefficients $F_{0}$ and $F_{1}$ are as follows:

$$
\begin{aligned}
F_{0}= & \frac{1}{k^{2} k !\left(-\left(A_{2}-2 k C_{1} / k^{2}\right)\right)^{k}} 2^{-1-k}\left(-\frac{A_{2}-2 k C_{1}}{k^{2}}\right)^{k} \\
& \left(-4 A_{2} k^{2}\left(5+4 k+3 k^{2}\right) C_{1}^{3}+4 k^{3}\left(3+3 k+3 k^{2}+k^{3}\right) C_{1}^{4}+8 k C_{1}^{2}\left(A_{2}(1+k)-k^{3}\left(2+3 k+2 k^{2}\right) C_{2}\right)\right. \\
& \left.-4\left(2 A_{2} k^{2} C_{2}-4(-1+k) k^{5} C_{2}^{2}+3 A_{2} k^{4} C_{3}\right)+C_{1}\left(-A_{2}^{3}+4 A_{2} k^{3}(4+7 k) C_{2}+24 k^{5} C_{3}\right)\right), \\
F_{1}= & \frac{1}{3 k^{3} k !} 2^{-k}\left(-\frac{A_{2}-2 k C_{1}}{k^{2}}\right)^{k}\left(3 C_{1}\left(A_{2} C_{1}-k(k+1) C_{1}^{2}+2 k^{2} C_{2}\right)+3 k C_{1}\left(A_{2} C_{1}-k(1+k) C_{1}^{2}+2 k^{2} C_{2}\right)\right. \\
& -\frac{4(-2+k)(-1+k) k\left(A_{2} C_{1}-k(1+k) C_{1}^{2}+2 k^{2} C_{2}\right)^{3}}{\left(A_{2}-2 k C_{1}\right)^{3}} \\
& +\frac{6(-1+k) k\left(A_{2} C_{1}-k(1+k) C_{1}^{2}+2 k^{2} C_{2}\right)\left(-A_{2}(3-2 k) C_{1}^{2}+2 k(1+k)^{2} C_{1}^{3}-4 A_{2} k C_{2}-2 k^{2}(4+3 k) C_{1} C_{2}-6 k^{3} C_{3}\right)}{\left(A_{2}-2 k C_{1}\right)^{2}} .
\end{aligned}
$$

From expressions (8) and (14), we obtain

$$
\begin{aligned}
t_{\zeta}= & -\frac{\left(A_{2}-2 k C_{1}\right)}{2 k^{2}} e_{\zeta}+\frac{\left(3 A_{2} C_{1}-2 k(2+k) C_{1}^{2}+4 k^{2} C_{2}\right)}{2 k^{3}} e_{\zeta}^{2} \\
& +\phi_{1} e_{\zeta}^{3}+\phi_{2} e_{\zeta}^{4}+O\left(e_{\zeta}^{5}\right),
\end{aligned}
$$

where $\quad \phi_{1}=\left(1 / 4 k^{5}\right)\left(-5 A_{2} k(3+k) C_{1}^{2}+2 k^{2}\left(7+7 k+2 k^{2}\right)\right.$ $\left.C_{1}^{3}+C_{1}\left(A_{2}^{2}-4 k^{3}(7+3 k) C_{2}\right)+2 k^{2}\left(5 A_{2} C_{2}+6 k^{2} C_{3}\right)\right), \phi_{2}=$ $\left(1 / 12 k^{6}\right)\left(A_{2} k\left(97-69 k-14 k^{2}\right) C_{1}^{3}-2 k^{2}\left(34+51 k+29 k^{2}+\right.\right.$ $\left.6 k^{3}\right) C_{1}^{4}+3 C_{1}^{2}\left(-5 A_{2}^{2}-4 k^{3}\left(17+16 k+4 k^{2}\right) C_{2}\right)-6 k^{2} C_{1}\left(A_{2}\right.$ $\left.(23+7 k) C_{2}+4 k^{2}(5+2 k) C_{3}\right)+6 k^{3}\left(-4 k(3+k) C_{2}^{2}-7 A_{2} C_{3}\right.$ $\left.+8 k^{2} C_{4}\right)$ ).

Expansion of the weight function $H\left(t_{\zeta}\right)$ by using Taylor series is given as follows:

$$
H\left(t_{\zeta}\right)=B_{0}+B_{1} t_{\zeta}+\frac{1}{2} B_{2} t_{\zeta}^{2}+\frac{1}{6} B_{3} t_{\zeta}^{3}
$$

where $B_{j}=\left(B^{j}(0) / j !\right)$ for $0 \leq j \leq 3$.

By inserting (11) and (17) in the second step of scheme (6), we attain 


$$
\left.\begin{array}{rl}
z_{\zeta}= & -B_{0} e_{\zeta}-\frac{1}{2 k^{2}}\left(\left(1+B_{0}-B_{1}\right)\left(A_{2}-2 k C_{1}\right)\right) e_{\zeta}^{2}+\frac{1}{8 k^{4}} \\
& \left(\begin{array}{c}
A_{2}^{2}\left(2 B_{1}-B_{2}\right)+4 A_{2}\left(2+2 B_{0}-5 B_{1}+B_{2}\right) k C_{1} \\
-4 k^{2}\left(2+B_{2}+2 k+2 B_{0}(1+k)-2 B_{1}(3+k)\right) C_{1}^{2}+16\left(1+B_{0}-B_{1}\right) k^{3} C_{2}
\end{array}\right) e_{\zeta}^{3} \\
& +\frac{1}{48 k^{6}}\left(\begin{array}{c}
C_{1}^{3}+24 A_{2}\left(4+4 B_{0}-9 B_{1}-2 B_{2}\right) k^{3} C_{2}-6 k C_{1} \\
C_{1}^{2}+8 k^{3}\left(6-15 B_{2}-B_{3}-6 B_{2} k+6 k^{2}+6 B_{0}(1+k)^{2}-3 B_{1}\left(13+11 k-2 k^{2}\right)\right) \\
\left(A_{2}^{2}\left(12 B_{1}-9 B_{2}+B_{3}\right)+8 k^{3}\left(4-11 B_{1}-2 B_{2}+3 k-3 B_{1} k+H(4+3 k)\right) C_{2}\right) \\
+144 k^{5} C_{3}-144 B_{0} k^{5} C_{3}-144 B_{1} k^{5} C_{3}
\end{array}\right)
\end{array}\right)
$$

By selecting $B_{0}=0, B_{1}=1, B_{2}=4$, and $A_{2}=0$, we get

$$
z_{\zeta}=\frac{1}{6 k^{3}}\left(\left(-B_{3}-3(9+k)\right) C_{1}^{3}-6 k C_{1} C_{2}\right) e_{\zeta}^{4}+O\left(e_{\zeta}^{5}\right) \text {. }
$$

Hence, scheme (6) reaches at fourth order of convergence by using its first two steps. obtain

Now, again by using the Taylor series expansion of $z_{\zeta}$, we

$$
\begin{aligned}
g\left(z_{\zeta}\right)= & g^{(k)}(\alpha) e^{4 k}\left(\frac{6^{-k}\left(\left(-B_{3}+3(9+k)\right) C_{1}^{3}-6 k C_{1} C_{2} / k^{3}\right)^{k}}{k !}+\frac{6^{-k}\left(\left(-B_{3}+3(9+k)\right) C_{1}^{3}-6 k C_{1} C_{2} / k^{3}\right)^{-1+k} \psi_{0}}{k^{3} k !}\right) \\
& +g^{(k)}(\alpha) e^{4 k}\left(\sum_{x=0}^{7} L_{x} e_{\zeta}^{x+1}\right)+O\left(e_{\zeta}^{9}\right),
\end{aligned}
$$

where $\psi_{0}=\left(-125-84 k-7 k^{2}+B_{3}(7+3 k)\right) C_{1}^{4}+6 k\left(-B_{3}+\right.$ $4(7+k)) C_{1}^{2} C_{2}-12 k^{2} C_{2}^{2}-12 k^{2} C_{1} C_{3}$.

With the help of expressions (14) and (20), we have

$$
s_{\zeta}=\frac{\left(\left(-B_{3}+3(9+k)\right) C_{1}^{2}-6 k C_{2}\right)}{6 k^{2}} e_{\zeta}^{2}+\psi_{1} e_{\zeta}^{3}+\psi_{2} e_{\zeta}^{4}+O\left(e_{\zeta}^{5}\right)
$$

where $\psi_{1}=\left(-49-27 k-2 k^{2}+B_{3}(3+k)\right) C_{1}^{3}+2 k\left(-B_{3}+3\right.$ $(9+k)) C_{1} C_{2}-\quad 6 k^{2} C_{3}, \quad \psi_{2}=\left(1 / 24 k^{4}\right)$ $\left(\begin{array}{c}\left(899+1002 k+313 k^{2}+18 k^{3}-2 B_{3}\left(43+33 k+6 k^{2}\right)\right) C_{1}^{4} \\ -4 k\left(501-33 B_{3}+261 k-10 B_{3} k+18 k^{2}\right) C_{1}^{2} C_{2}+24 k^{2}\left(26-B_{3}-3 k\right) C_{1} C_{3} \\ +4 k^{2}\left(\left(105-4 B_{3}-9 k\right) C_{2}^{2}-18 k C_{4}\right)\end{array}\right)$.

With the help of expressions (8) and (20), we obtain $w_{\zeta}=\frac{1}{6 k^{3}}\left(\left(-B_{3}+3(9+k)\right) C_{1}^{2}-6 k C_{1} C_{2}\right) e_{\zeta}^{3}+\psi_{3} e_{\zeta}^{4}+O\left(e_{\zeta}^{5}\right)$, where $\quad \psi_{3}=\left(1 / 6 k^{4}\right)\left(\left(-152-87 k-7 k^{2}+B_{3}(8+3 k)\right) C_{1}^{4}+\right.$ $\left.6 k\left(29-B_{3}+4 k\right) C_{1}^{2} C_{2}-12 k^{2} C_{2}^{2}-12 k^{2} C_{1} C_{3}\right)$.

Now, we expand the trivariate weight function $D\left(t_{\zeta}, s_{\zeta}, w_{\zeta}\right)$ in the neighborhood of $(0,0,0)$ by using the Taylor series expansion as follows:

$$
\begin{aligned}
D\left(t_{\zeta}, s_{\zeta}, w_{\zeta}\right)= & D_{000}+D_{100} t_{\zeta}+D_{010} s_{\zeta}+D_{001} w_{\zeta}+D_{101} t_{\zeta} w_{\zeta} \\
& +D_{011} s_{\zeta} w_{\zeta}+D_{111} t_{\zeta} s_{\zeta} w_{\zeta}+\frac{1}{2} D_{200} t_{\zeta}^{2} \\
& +\frac{1}{2} D_{020} s_{\zeta}^{2}+\frac{1}{2} D_{002} w_{\zeta}^{2}
\end{aligned}
$$

where $D_{i j k}=(1 / i ! j ! x k !)\left(\partial^{i+j+k} / \partial t_{\zeta}^{i} \partial s_{\zeta}^{j} \partial w_{\zeta}^{x}\right) D\left(t_{\zeta}, s_{\zeta}, w_{\zeta}\right)$, for $i, j, k \in \mathbb{N}$. 
We obtain the asymptotic error constant term by employing expressions (11), (17), and (23) in the third step of scheme (6), as follows:

$$
\begin{aligned}
e_{\zeta+1}= & \frac{D_{000} C_{1}}{k} e_{\zeta}^{2}+\frac{\left(\left(D_{000}+k D_{000}-D_{100}\right) C_{1}^{2}-2 k D_{000} C_{2}\right)}{k^{2}} e_{\zeta}^{3} \\
& +D_{4} e_{\zeta}^{4}+D_{5} e_{\zeta}^{5}+D_{6} e_{\zeta}^{6}+D_{7} e_{\zeta}^{7}+O\left(e_{\zeta}^{8}\right) .
\end{aligned}
$$

The coefficients $D_{i}(4 \leq i \leq 7)$ simultaneously depend generally upon the weights $D_{i j k}$ and multiplicity $k$. To obtain the eighth order convergence of scheme (6), we have to select the following conditions of the weights such as $D_{000}=0$; $D_{100}=0 ; D_{010}=1 ; D_{200}=0 ; D_{001}=-1 ; D_{110}=1 ; D_{020}=2$; $D_{101}=1 ; D_{011}=2$; and $B_{3}=18$.

By using the above conditions, we get the required asymptotic error constant equation as follows:

$$
e_{\zeta+1}=\frac{1}{24 k^{7}}\left\{C_{1}\left((3+k) C_{1}^{2}-2 k C_{2}\right)\left(\left(-163+7 k^{2}\right) C_{1}^{4}-24 k^{2} C_{1}^{2} C_{2}-12 k^{2} C_{2}^{2}+12 k^{2} C_{1} C_{3}\right) e_{\zeta}^{8}\right\}+O\left(e_{\zeta}^{9}\right)
$$

The above equation (25) confirms that the proposed scheme (6) has optimal eighth order of convergence consuming only one-derivative and three-function evaluations per full iteration (i.e., $g^{\prime}\left(x_{\zeta}\right), g\left(x_{\zeta}\right), g\left(y_{\zeta}\right)$ and $\left.g\left(z_{\zeta}\right)\right)$.

\section{Some Special Cases of Weight Functions}

We consider some special choices of weight functions employed in our new scheme (6) satisfying the conditions of Theorem 1. The considered weight functions are simple.

First, we consider the polynomial weight function $G\left(v_{\zeta}\right)$, satisfying the conditions given in Theorem 1 and can be represented as

$$
G\left(v_{\zeta}\right)=k \cdot v_{\zeta}, \quad \zeta \geq 0
$$

The trivariate weight function $D\left(t_{\zeta}, s_{\zeta}, w_{\zeta}\right)$ is also chosen as a polynomial and is given as

$$
D\left(t_{\zeta}, s_{\zeta}, w_{\zeta}\right)=s_{\zeta} \cdot\left(1+s_{\zeta}+2 s_{\zeta} t_{\zeta}+t_{\zeta}^{2}\right)+D_{201} \cdot w_{\zeta} t_{\zeta}^{2}
$$

where $D_{201}$ is a free parameter. By taking different choices for $D_{201}$, we have three cases described as follows.

3.1. Case 1. We take the polynomial weight function $H\left(t_{\zeta}\right)$ directly from Theorem 1 as follows:

$$
H\left(t_{\zeta}\right)=t_{\zeta}+2 t_{\zeta}^{2}
$$

We select the parameter $D_{201}=-6$ in (28), and also by using the weight functions given in equations (26), (28), and (27), respectively, we prevail the special case of our newly developed optimal eighth order family (6), named as SFM1 given by

$$
\begin{aligned}
y_{\zeta} & =e_{\zeta}-\left(k \cdot v_{\zeta}\right), \quad \zeta \geq 0, \\
z_{\zeta} & =y_{\zeta}-\left(t_{\zeta}+2 t_{\zeta}^{2}\right) \times\left(k \cdot v_{\zeta}\right), \\
x_{\zeta+1} & =z_{\zeta}-\left(t_{\zeta}+2 t_{\zeta}^{2}\right) \times\left(s_{\zeta} \cdot\left(1+s_{\zeta}+2 s_{\zeta} t_{\zeta}+t_{\zeta}^{2}\right)-6 w_{\zeta} t_{\zeta}^{2}\right) \times\left(k \cdot v_{\zeta}\right),
\end{aligned}
$$

where $v_{\zeta}=\left(g\left(x_{\zeta}\right) / g^{\prime}\left(x_{\zeta}\right)\right), t_{\zeta}=\left[\left(g\left(y_{\zeta}\right) / g\left(x_{\zeta}\right)\right)\right]^{(1 / k)}, s \quad \zeta=$ $\left[\left(g\left(z_{\zeta}\right) / g\left(y_{\zeta}\right)\right)\right]^{(1 / k)}, w_{\zeta}=\left[\left(g\left(z_{\zeta}\right) / g\left(x_{\zeta}\right)\right)\right]^{(1 / k)}$.

3.2. Case 2. By keeping the fact in our mind that the rational functions show more stable behavior by using the same degree polynomial in contrast to simple polynomial of the same degree, we take the rational weight function $H\left(t_{\zeta}\right)$ from the hypothesis of Theorem 1 as follows:

$$
H\left(t_{\zeta}\right)=\frac{t_{\zeta}}{1-2 t_{\zeta}} .
$$

For this special choice of weight function given in (30), we choose the parameter $D_{201}=2$, involved in expression (27). By using the weight functions given in equations (26), (30), and (27), respectively, we obtain another special case of our newly developed optimal eighth order family (6) denoted by SFM2 as follows: 


$$
\begin{aligned}
& y_{\zeta}=e_{\zeta}-\left(k \cdot v_{\zeta}\right), \quad \zeta \geq 0, \\
& z_{\zeta}=y_{\zeta}-\left(\frac{t_{\zeta}}{1-2 t_{\zeta}}\right) \times\left(k \cdot v_{\zeta}\right), \\
& x_{\zeta+1}=z_{\zeta}-\left(\frac{t_{\zeta}}{1-2 t_{\zeta}}\right) \times\left(s_{\zeta} \cdot\left(1+s_{\zeta}+2 s_{\zeta} t_{\zeta}+t_{\zeta}^{2}\right)+2 w_{\zeta} t_{\zeta}^{2}\right) \times\left(k \cdot v_{\zeta}\right), \\
& \left.g^{\prime}\left(x_{\zeta}\right)\right), t_{\zeta}=\left[\left(g\left(y_{\zeta}\right) / g\left(x_{\zeta}\right)\right)\right]^{(1 / k)}, \quad H\left(t_{\zeta}\right)=\frac{5}{2} t_{\zeta}^{2}+\log \left(t_{\zeta}+1\right) .
\end{aligned}
$$

where $\quad v_{\zeta}=\left(g\left(x_{\zeta}\right) / g^{\prime}\left(x_{\zeta}\right)\right), t_{\zeta}=\left[\left(g\left(y_{\zeta}\right) / g\left(x_{\zeta}\right)\right)\right]^{(1 / k)}$, $s_{\zeta}=\left[\left(g\left(z_{\zeta}\right) / g\left(y_{\zeta}\right)\right)\right]^{(1 / k)}, w_{\zeta}=\left[\left(g\left(z_{\zeta}\right) / g\left(x_{\zeta}\right)\right)\right]^{(1 / k)}$.

3.3. Case 3. We consider the logarithmic weight function $H\left(t_{\zeta}\right)$ as follows:
By using $D_{201}=-(16 / 3)$ in (27) and the weight functions given in equations (26), (32), and (27), respectively, we prevail the special case of the family (6), named as SFM3, given as

$$
\begin{aligned}
y_{\zeta} & =e_{\zeta}-\left(k \cdot v_{\zeta}\right), \quad \zeta \geq 0, \\
z_{\zeta} & =y_{\zeta}-\left(\frac{5}{2} t_{\zeta}^{2}+\log \left(t_{\zeta}+1\right)\right) \times\left(k \cdot v_{\zeta}\right), \\
x_{\zeta+1} & =z_{\zeta}-\left(\frac{5}{2} t_{\zeta}^{2}+\log \left(t_{\zeta}+1\right)\right) \times\left(s_{\zeta} \cdot\left(1+s_{\zeta}+2 s_{\zeta} t_{\zeta}+t_{\zeta}^{2}\right)-\frac{16}{3} w_{\zeta} t_{\zeta}^{2}\right) \times\left(k \cdot v_{\zeta}\right),
\end{aligned}
$$

where $v_{\zeta}=\left(g\left(x_{\zeta}\right) / g^{\prime}\left(x_{\zeta}\right)\right), t_{\zeta}=\left[\left(g\left(y_{\zeta}\right) / g\left(x_{\zeta}\right)\right)\right]^{(1 / k)}, s_{\zeta}=[$ $\left.\left(g\left(z_{\zeta}\right) / g\left(y_{\zeta}\right)\right)\right]^{(1 / k)}, w_{\zeta}=\left[\left(g\left(z_{\zeta}\right) / g\left(x_{\zeta}\right)\right)\right]^{(1 / k)}$.

\section{Computational Results}

In this section, we examine the convergence behavior, strength, and effectiveness of the proposed multiple root finding scheme (6). We consider some numerical test functions including two real-life problems and some standard nonlinear functions. We take the special cases of our newly proposed scheme given in (29), (31), and (33) denoted by SFM1, SFM2, and SFM3, respectively. We compare them with the eighth order optimal scheme by Behl et al. [18], given in (3). We choose a special case of their scheme with the parametric values $\left(\beta_{1}=1\right.$ and $\left.\beta_{2}=1\right)$ given below, denoted by BM1:

$$
\begin{aligned}
& y_{\zeta}=x_{\zeta}-k \cdot \frac{g\left(x_{\zeta}\right)}{g^{\prime}\left(x_{\zeta}\right)}, \quad \zeta \geq 0, \\
& z_{\zeta}=y_{\zeta}-\left(k+2 t_{\zeta} k \beta_{1}+\frac{1}{2} t_{\zeta}^{2}\left(4 k \beta_{1}^{2}+2 k \beta_{1} \beta_{2}\right)\right) \cdot s_{\zeta} \cdot \frac{g\left(x_{\zeta}\right)}{g^{\prime}\left(x_{\zeta}\right)}, \\
& x_{\zeta+1}=z_{\zeta}-\left(\frac{k\left(1+2 w_{\zeta}+3 t_{\zeta}^{2} \beta_{1}^{2}+t_{\zeta} \beta_{1}\left(2+6 w_{\zeta}+t_{\zeta} \beta_{2}\right)\right)}{1+w_{\zeta}}\right) \cdot s_{\zeta} \cdot w_{\zeta} \cdot \frac{g\left(x_{\zeta}\right)}{g^{\prime}\left(x_{\zeta}\right)},
\end{aligned}
$$


where $\quad s_{\zeta}=\left[\left(g\left(y_{\zeta}\right) / g\left(x_{\zeta}\right)\right)\right]^{(1 / k)}, t_{\zeta}=\left(s_{\zeta} / \beta_{1}+\beta_{2} s_{\zeta}\right), w_{\zeta}=$ $\left[\left(g\left(z_{\zeta}\right) / g\left(y_{\zeta}\right)\right)\right]^{(1 / k)}$.

We also compare the results of our methods with the three-step optimal eighth order family by Akram et al. [19], given in (4). We choose the following special case of their scheme denoted by AM2:

$$
\begin{gathered}
y_{\zeta}=x_{\zeta}-k \cdot \frac{g\left(x_{\zeta}\right)}{g^{\prime}\left(x_{\zeta}\right)}, \quad \zeta \geq 0, \\
z_{\zeta}=y_{\zeta}-k \cdot\left(6 s_{\zeta}^{3}-s_{\zeta}^{2}+2 s_{\zeta}+1\right) \cdot s_{\zeta} \cdot \frac{g\left(x_{\zeta}\right)}{g^{\prime}\left(x_{\zeta}\right)}, \\
x_{\zeta+1}=z_{\zeta}-k \cdot\left(2 w_{\zeta}+4 t_{\zeta} w_{\zeta}+t_{\zeta}+t_{\zeta}^{2}\right) \cdot s_{\zeta} \cdot \frac{g\left(x_{\zeta}\right)}{g^{\prime}\left(x_{\zeta}\right)},
\end{gathered}
$$

where $s_{\zeta}=\left[\left(g\left(y_{\zeta}\right) / g\left(x_{\zeta}\right)\right)\right]^{(1 / k)}, t_{\zeta}=\left[\left(g\left(z_{\zeta}\right) / g\left(y_{\zeta}\right)\right)\right]^{(1 / k)}$, $w_{\zeta}=\left[\left(g\left(z_{\zeta}\right) / g\left(x_{\zeta}\right)\right)\right]^{(1 / k)}$.

We compare our methods with another three-step optimal eighth order family presented by Zafar et al. [20], given in (5). We select the following special case of their scheme denoted by ZM3:

$$
\begin{aligned}
& y_{\zeta}=x_{\zeta}-k \cdot \frac{g\left(x_{\zeta}\right)}{g^{\prime}\left(x_{\zeta}\right)}, \quad \zeta \geq 0, \\
& z_{\zeta}=y_{\zeta}-k \cdot\left(1+6 s_{\zeta}^{3}-s_{\zeta}^{2}+2 s_{\zeta}\right) \cdot s_{\zeta} \cdot \frac{g\left(x_{\zeta}\right)}{g^{\prime}\left(x_{\zeta}\right)}, \\
& x_{\zeta+1}=z_{\zeta}-k \cdot\left(2 t_{\zeta}+1\right) \cdot\left(w_{\zeta}+1\right) \cdot\left(2 s_{\zeta}+1\right) \cdot s_{\zeta} \cdot w_{\zeta} \cdot \frac{g\left(x_{\zeta}\right)}{g^{\prime}\left(x_{\zeta}\right)},
\end{aligned}
$$

where $s_{\zeta}=\left[\left(g\left(y_{\zeta}\right) / g\left(x_{\zeta}\right)\right)\right]^{(1 / k)}, w_{\zeta}=\left[\left(g\left(z_{\zeta}\right) / g\left(y_{\zeta}\right)\right)\right]^{(1 / k)}$, $t_{\zeta}=\left[\left(g\left(z_{\zeta}\right) / g\left(x_{\zeta}\right)\right)\right]^{(1 / k)}$.

Comparison has been made on the basis of $\left|g\left(x_{\zeta}\right)\right|$, that is, function value at $x_{\zeta}$ or also known as the absolute residual error, $\left|x_{\zeta}-\alpha\right|$, that is, the absolute error approximation to the sought zero at each iteration, $\mathrm{AEC}=\mid\left(x_{\zeta+1}-x_{\zeta}\right.$ $\left.\mid\left(x_{\zeta}-x_{\zeta-1}\right)^{r}\right) \mid(r$ represents the order of convergence), that is, the asymptotic error constant and $\mathrm{COC} \approx\left(\log \mid\left(x_{\zeta+1}-\right.\right.$ $\left.\alpha) /\left(x_{\zeta}-\alpha\right)|/ \log |\left(x_{\zeta}-\alpha\right) /\left(x_{\zeta-1}-\alpha\right) \mid\right)$ (formula given by Jay [21]), that is, the computational order of convergence. Tables 1-7 show the comparison of the methods BM1, AM2, and ZM3, given in (34), (35), and (36), with our newly developed methods SFM1, SFM2, and SFM3. The programming packages Maple 18 and Wolfram Mathematica 8 have been used to perform all the computational works for the numerical and theoretical results. The calculations for numerical results have been made by using several (minimum 1000) number of significant digits of precision. Due to limited space of paper, numerical results are displayed up to only four decimal places.

Example 1. Van der Waals' equation of state
Van der Waals' equation is particularly useful in our effort to understand the behavior of real gases, because it embodies a simple physical picture for the difference between a real and an ideal gas. We know the ideal gas law as

$$
\mathrm{PV}=n R T \text {. }
$$

The relationship between the pressure $P$, the volume $V$, and the temperature $T$ for real gases is the Van der Waals' equation expressed as

$$
\left(P+\frac{\beta_{1} n^{2}}{V^{2}}\right)\left(V-n \beta_{2}\right)=n R T .
$$

This expression explains the behavior of real gas by taking in the ideal gas equation. The involving two parameters $\beta_{1}$ and $\beta_{2}$ are specific for each gas. We require the solution of the following nonlinear function in $V$ to determine the volume $V$ of the gas in terms of the remaining parameters:

$$
\mathrm{PV}^{3}-\left(n \beta_{2} P+n R T\right) V^{2}+\beta_{1} n^{2} V-\beta_{1} \beta_{2} n^{3}=0 .
$$

Considering the involving parameters $\beta_{1}$ and $\beta_{2}$ of a particular gas, one can find values for $n, P$, and $T$. By the implementation of particular values, we have the below given nonlinear function:

$$
g_{1}(x)=x^{3}-5.22 x^{2}+9.0825 x-5.2675
$$

where $g_{1}(x)$ has three roots. One is simple root $\alpha=1.72$ and the other is a multiple zero $\alpha=1.75$ of multiplicity 2 . We considered the initial approximation $x_{0}=1.8$ for this test problem. The computational results are mentioned in Table 1.

Regarding the results shown in Table 1, the convergence behavior of newly developed methods SFM1, SFM2, and SFM3 is far better than the earlier known methods BM1, $\mathrm{AM} 2$, and ZM3 for the test problem $g_{1}(x)$. The comparison of function value at $x_{\zeta}$ and absolute error shows that SFM2 and SFM3 have smaller error values than the existing known methods.

Example 2. Beam designing model.

We consider a beam positioning problem [22] where a beam of $p$ unit length is straight towards the edge of the cubical box having 1 unit length of each side. One end of the beam touches the wall and the other end touches with the floor, as depicted in Figure 1.

We have problem here to measure the distance alongside the floor from the base of the wall to the bottom of the beam. Assume that $x$ is the distance in units from the bottom of the beam at floor to the bottom of the box and let $y$ be the distance in units from the bottom of the beam at floor to the edge of the box, along the beam. Then, by employing a specific value of $p$, we have the following nonlinear equation:

$$
g_{2}(x)=x^{4}+4 x^{3}-24 x^{2}+16 x+16=0,
$$

having a multiple zero $\alpha=2.0$ of multiplicity 2 . We considered the initial approximation $x_{0}=2.5$ for this test function. The comparison results are depicted in Table 2. 
TABle 1: Convergence behavior of different iterative methods for $g_{1}(x)$.

\begin{tabular}{|c|c|c|c|c|c|c|}
\hline \multicolumn{7}{|c|}{$g_{1}(x)=x^{3}-5.22 x^{2}+9.0825 x-5.2675, x_{0}=1.8$} \\
\hline & $\zeta$ & $x_{\zeta}$ & $\left|g\left(x_{\zeta}\right)\right|$ & $\left|x_{\zeta}-\alpha\right|$ & AEC & $\mathrm{COC}$ \\
\hline \multirow{3}{*}{ BM1 } & 1 & 1.75 & $9.9347 \times 10^{-9}$ & $5.7007 \times 10^{-4}$ & & \\
\hline & 2 & 1.75 & $5.5189 \times 10^{-32}$ & $1.3563 \times 10^{-15}$ & $7.9067 \times 10^{5}$ & \\
\hline & 3 & 1.75 & $8.4215 \times 10^{-218}$ & $1.6754 \times 10^{-108}$ & $6.9320 \times 10^{7}$ & 7.990 \\
\hline \multirow{3}{*}{ AM2 } & 1 & 1.75 & $6.6085 \times 10^{-9}$ & $4.6574 \times 10^{-4}$ & & \\
\hline & 2 & 1.75 & $3.1520 \times 10^{-33}$ & $3.2414 \times 10^{-16}$ & $1.2849 \times 10^{7}$ & \\
\hline & 3 & 1.75 & $1.4151 \times 10^{-227}$ & $2.1718 \times 10^{-113}$ & $1.464 \times 10^{11}$ & 7.990 \\
\hline \multirow{3}{*}{ ZM3 } & 1 & 1.75 & $4.5788 \times 10^{-9}$ & $3.8817 \times 10^{-4}$ & & \\
\hline & 2 & 1.75 & $7.9903 \times 10^{-35}$ & $5.1608 \times 10^{-17}$ & $1.057 \times 10^{7}$ & \\
\hline & 3 & 1.75 & $1.0549 \times 10^{-240}$ & $5.9301 \times 10^{-120}$ & $1.001 \times 10^{11}$ & 7.992 \\
\hline \multirow{3}{*}{ SFM1 } & 1 & 1.75 & $5.5235 \times 10^{-9}$ & $4.2607 \times 10^{-4}$ & & \\
\hline & 2 & 1.75 & $2.0673 \times 10^{-34}$ & $8.3013 \times 10^{-17}$ & $1.1680 \times 10^{7}$ & \\
\hline & 3 & 1.75 & $1.2060 \times 10^{-237}$ & $2.0050 \times 10^{-118}$ & $7.6431 \times 10^{10}$ & 7.992 \\
\hline \multirow{3}{*}{ SFM2 } & 1 & 1.75 & $1.0796 \times 10^{-9}$ & $1.8910 \times 10^{-4}$ & & \\
\hline & 2 & 1.75 & $3.6782 \times 10^{-42}$ & $1.1072 \times 10^{-20}$ & $4.990 \times 10^{6}$ & \\
\hline & 3 & 1.75 & $7.7223 \times 10^{-302}$ & $1.6044 \times 10^{-150}$ & $6.7693 \times 10^{9}$ & 7.998 \\
\hline \multirow{3}{*}{ SFM3 } & 1 & 1.75 & $5.0786 \times 10^{-9}$ & $4.0867 \times 10^{-4}$ & & \\
\hline & 2 & 1.75 & $7.3241 \times 10^{-35}$ & $4.9410 \times 10^{-17}$ & $1.117 \times 10^{7}$ & \\
\hline & 3 & 1.75 & $2.0151 \times 10^{-241}$ & $2.5917 \times 10^{-120}$ & $6.350 \times 10^{10}$ & 7.993 \\
\hline
\end{tabular}

TABle 2: Convergence behavior of different iterative methods for $g_{2}(x)$.

\begin{tabular}{|c|c|c|c|c|c|c|}
\hline \multicolumn{7}{|c|}{$g_{2}(x)=x^{4}+4 x^{3}-24 x^{2}+16 x+16, x_{0}=2.5$} \\
\hline & $\zeta$ & $x_{\zeta}$ & $\left|g\left(x_{\zeta}\right)\right|$ & $\left|x_{\zeta}-\alpha\right|$ & AEC & $\mathrm{COC}$ \\
\hline \multirow{3}{*}{ BM1 } & 1 & 2.0 & $3.2691 \times 10^{-9}$ & $1.1671 \times 10^{-5}$ & & \\
\hline & 2 & 2.0 & $1.1447 \times 10^{-81}$ & $6.9063 \times 10^{-42}$ & $1.494 \times 10^{-3}$ & \\
\hline & 3 & 2.0 & $2.5882 \times 10^{-661}$ & $1.0384 \times 10^{-331}$ & $2.341 \times 10^{-7}$ & 7.99 \\
\hline \multirow{3}{*}{ AM2 } & 1 & 2.0 & $2.4815 \times 10^{-9}$ & $1.0168 \times 10^{-5}$ & & \\
\hline & 2 & 2.0 & $2.1471 \times 10^{-82}$ & $2.9910 \times 10^{-42}$ & $2.603 \times 10^{-3}$ & \\
\hline & 3 & 2.0 & $6.7449 \times 10^{-667}$ & $1.6764 \times 10^{-334}$ & $2.616 \times 10^{-2}$ & 7.99 \\
\hline \multirow{3}{*}{ ZM3 } & 1 & 2.0 & $1.1173 \times 10^{-9}$ & $6.8232 \times 10^{-6}$ & & \\
\hline & 2 & 2.0 & $1.5102 \times 10^{-85}$ & $7.9327 \times 10^{-44}$ & $1.746 \times 10^{-3}$ & \\
\hline & 3 & 2.0 & $1.6824 \times 10^{-692}$ & $2.6477 \times 10^{-347}$ & $1.688 \times 10^{-2}$ & 7.99 \\
\hline \multirow{3}{*}{ SFM1 } & 1 & 2.0 & $1.1137 \times 10^{-9}$ & $6.8121 \times 10^{-6}$ & & \\
\hline & 2 & 2.0 & $8.0859 \times 10^{-86}$ & $5.8044 \times 10^{-44}$ & $1.744 \times 10^{-3}$ & \\
\hline & 3 & 2.0 & $6.2434 \times 10^{-695}$ & $1.6128 \times 10^{-348}$ & $1.251 \times 10^{-2}$ & 7.99 \\
\hline \multirow{3}{*}{ SFM2 } & 1 & 2.0 & $1.2487 \times 10^{-11}$ & $7.2132 \times 10^{-7}$ & & \\
\hline & 2 & 2.0 & $7.0178 \times 10^{-104}$ & $5.4074 \times 10^{-53}$ & $1.846 \times 10^{-4}$ & \\
\hline & 3 & 2.0 & $6.9822 \times 10^{-842}$ & $5.3937 \times 10^{-422}$ & $7.377 \times 10^{-4}$ & 7.99 \\
\hline \multirow{3}{*}{ SFM3 } & 1 & 2.0 & $8.4488 \times 10^{-10}$ & $5.9332 \times 10^{-6}$ & & \\
\hline & 2 & 2.0 & $5.7599 \times 10^{-87}$ & $1.5491 \times 10^{-44}$ & $1.519 \times 10^{-3}$ & \\
\hline & 3 & 2.0 & $2.6878 \times 10^{-704}$ & $3.3465 \times 10^{-353}$ & $1.008 \times 10^{-2}$ & 7.99 \\
\hline
\end{tabular}

The comparison results of the model presented in Table 2 show the newly developed schemes SFM1, SFM2, and SFM3 are not only convergent but also their speed of convergence is better than BM1, AM2, and ZM3.

Example 3. Further, we consider the standard test problem involving trigonometric function is as follows:

$$
g_{3}(x)=\left(\sin ^{2} x+x\right)^{5}
$$

having a root $\alpha=0.0$ of multiplicity 5 . We considered the initial approximation $x_{0}=0.2$ for this test problem. The numerical results are shown in Table 3.

In Table 3, it can be seen that the newly proposed methods SFM2 and SFM3 are computationally more efficient than all other methods BM1, AM2, and ZM3 for the test function $g_{3}(x)$. Among all the methods, convergence behavior of SFM2 is very efficient and results of SFM1 are comparable with ZM3. 
TABle 3: Convergence behavior of different iterative methods for $g_{3}(x)$.

\begin{tabular}{|c|c|c|c|c|c|c|}
\hline \multicolumn{7}{|c|}{$g_{3}(x)=\left(\sin ^{2} x+x\right)^{5}, x_{0}=0.2$} \\
\hline & $\zeta$ & $x_{\zeta}$ & $\left|g\left(x_{\zeta}\right)\right|$ & $\left|x_{\zeta}-\alpha\right|$ & AEC & $\mathrm{COC}$ \\
\hline \multirow{3}{*}{ BM1 } & 1 & 0.0 & $2.7742 \times 10^{-22}$ & $4.8821 \times 10^{-5}$ & & \\
\hline & 2 & 0.0 & $7.2420 \times 10^{-161}$ & $9.3750 \times 10^{-33}$ & 3.820 & \\
\hline & 3 & 0.0 & $1.5699 \times 10^{-1269}$ & $1.7345 \times 10^{-254}$ & 0.014 & 7.99 \\
\hline \multirow{3}{*}{ AM2 } & 1 & 0.0 & $9.2496 \times 10^{-23}$ & $3.9192 \times 10^{-5}$ & & \\
\hline & 2 & 0.0 & $5.0593 \times 10^{-164}$ & $2.1918 \times 10^{-33}$ & 15.333 & \\
\hline & 3 & 0.0 & $4.0762 \times 10^{-1294}$ & $2.0991 \times 10^{-259}$ & 393.69 & 7.99 \\
\hline \multirow{3}{*}{ ZM3 } & 1 & 0.0 & $1.4220 \times 10^{-23}$ & $2.6950 \times 10^{-5}$ & & \\
\hline & 2 & 0.0 & $1.6273 \times 10^{-171}$ & $6.9550 \times 10^{-35}$ & 10.539 & \\
\hline & 3 & 0.0 & $4.8042 \times 10^{-1355}$ & $1.3687 \times 10^{-271}$ & 249.86 & 7.99 \\
\hline \multirow{3}{*}{ SFM1 } & 1 & 0.0 & $2.04471 \times 10^{-23}$ & $2.8981 \times 10^{-5}$ & & \\
\hline & 2 & 0.0 & $6.3062 \times 10^{-171}$ & $9.1191 \times 10^{-35}$ & 11.333 & \\
\hline & 3 & 0.0 & $5.1803 \times 10^{-1351}$ & $8.7674 \times 10^{-271}$ & 183.24 & 7.99 \\
\hline \multirow{3}{*}{ SFM2 } & 1 & 0.0 & $9.0702 \times 10^{-28}$ & $3.9041 \times 10^{-6}$ & & \\
\hline & 2 & 0.0 & $2.2390 \times 10^{-212}$ & $4.6775 \times 10^{-43}$ & 1.5252 & \\
\hline & 3 & 0.0 & $3.0889 \times 10^{-1689}$ & $1.9859 \times 10^{-338}$ & 8.6663 & 7.99 \\
\hline \multirow{3}{*}{ SFM3 } & 1 & 0.0 & $1.1580 \times 10^{-23}$ & $2.5866 \times 10^{-5}$ & & \\
\hline & 2 & 0.0 & $2.1367 \times 10^{-173}$ & $2.9238 \times 10^{-35}$ & 10.114 & \\
\hline & 3 & 0.0 & $2.8788 \times 10^{-1371}$ & $7.7954 \times 10^{-275}$ & 145.90 & 7.99 \\
\hline
\end{tabular}

TABLE 4: Convergence behavior of different iterative methods for $g_{4}(x)$.

\begin{tabular}{|c|c|c|c|c|c|c|}
\hline \multicolumn{7}{|c|}{$g_{4}(x)=(x-1)\left(x \ln (x)-\sqrt{x}+x^{4}\right)^{2}, x_{0}=1.2$} \\
\hline & $\zeta$ & $x_{\zeta}$ & $\left|g\left(x_{\zeta}\right)\right|$ & $\left|x_{\zeta}-\alpha\right|$ & AEC & $\mathrm{COC}$ \\
\hline \multirow{3}{*}{ BM1 } & 1 & 1.0 & $5.0023 \times 10^{-12}$ & $6.2741 \times 10^{-5}$ & & \\
\hline & 2 & 1.0 & $2.2328 \times 10^{-93}$ & $4.7952 \times 10^{-32}$ & 4.9124 & \\
\hline & 3 & 1.0 & $3.5294 \times 10^{-744}$ & $3.5294 \times 10^{-249}$ & 0.0125 & 7.99 \\
\hline \multirow{3}{*}{ AM2 } & 1 & 1.0 & $2.8876 \times 10^{-12}$ & $5.2241 \times 10^{-5}$ & & \\
\hline & 2 & 1.0 & $8.9735 \times 10^{-95}$ & $1.6425 \times 10^{-32}$ & 20.449 & \\
\hline & 3 & 1.0 & $7.8338 \times 10^{-755}$ & $1.5698 \times 10^{-252}$ & 296.052 & 7.99 \\
\hline \multirow{3}{*}{ ZM3 } & 1 & 1.0 & $8.1638 \times 10^{-13}$ & $3.4287 \times 10^{-5}$ & & \\
\hline & 2 & 1.0 & $8.7066 \times 10^{-100}$ & $3.5032 \times 10^{-34}$ & 13.412 & \\
\hline & 3 & 1.0 & $1.4607 \times 10^{-795}$ & $4.1628 \times 10^{-266}$ & 183.375 & 7.99 \\
\hline \multirow{3}{*}{ SFM1 } & 1 & 1.0 & $1.0911 \times 10^{-12}$ & $3.7768 \times 10^{-5}$ & & \\
\hline & 2 & 1.0 & $3.3006 \times 10^{-99}$ & $5.4625 \times 10^{-34}$ & 14.775 & \\
\hline & 3 & 1.0 & $2.3195 \times 10^{-791}$ & $1.0463 \times 10^{-264}$ & 131.923 & 7.99 \\
\hline \multirow{3}{*}{ SFM2 } & 1 & 1.0 & $3.7040 \times 10^{-16}$ & $2.6348 \times 10^{-6}$ & & \\
\hline & 2 & 1.0 & $1.6585 \times 10^{-131}$ & $9.3563 \times 10^{-45}$ & 1.0293 & \\
\hline & 3 & 1.0 & $2.6810 \times 10^{-1054}$ & $2.3656 \times 10^{-352}$ & 4.0281 & 7.99 \\
\hline \multirow{3}{*}{ SFM3 } & 1 & 1.0 & $7.2300 \times 10^{-13}$ & $3.2927 \times 10^{-5}$ & & \\
\hline & 2 & 1.0 & $5.8147 \times 10^{-101}$ & $1.4213 \times 10^{-34}$ & 12.879 & \\
\hline & 3 & 1.0 & $1.0197 \times 10^{-805}$ & $1.7140 \times 10^{-269}$ & 102.858 & 7.99 \\
\hline
\end{tabular}

Example 4. Now, we choose standard nonlinear problem involving logarithmic function, which is given as

$$
g_{4}(x)=(x-1)\left(x \ln (x)-\sqrt{x}+x^{4}\right)^{2}
$$

having a root $\alpha=1.0$ of multiplicity 3 . We considered the initial approximation $x_{0}=1.2$ for this test problem. The comparison results are depicted in Table 4.

In Table 4, we can see that the newly developed method performs better than the existing presented schemes BM1, AM2, and ZM3. However, ZM3 performs slightly better than
SFM1, for the test function $g_{4}(x)$. SFM2 performs very well among all the presented methods.

Example 5. We consider one more standard nonlinear test problem, which is as follows:

$$
g_{5}(x)=\left(x^{3}-2 x^{2}+1\right)^{3}
$$

We select $\alpha=1.0$ multiple zero of multiplicity 6 for the computational point of view. The comparison results are shown in Table 5 corresponding to initial approximation $x_{0}=1.2$. 
TABle 5: Convergence behavior of different iterative methods for $g_{5}(x)$.

\begin{tabular}{|c|c|c|c|c|c|c|}
\hline \multicolumn{7}{|c|}{$g_{5}(x)=\left(x^{3}-2 x^{2}+1\right)^{3}, x_{0}=1.2$} \\
\hline & $\zeta$ & $x_{\zeta}$ & $\left|g\left(x_{\zeta}\right)\right|$ & $\left|x_{\zeta}-\alpha\right|$ & AEC & $\mathrm{COC}$ \\
\hline \multirow{3}{*}{ BM1 } & 1 & 1.0 & $6.0797 \times 10^{-34}$ & $1.4552 \times 10^{-6}$ & & \\
\hline & 2 & 1.0 & $5.9820 \times 10^{-271}$ & $4.5896 \times 10^{-47}$ & 0.113 & \\
\hline & 3 & 1.0 & $5.2548 \times 10^{-2221}$ & $4.4915 \times 10^{-371}$ & 0.003 & 7.99 \\
\hline \multirow{3}{*}{ AM2 } & 1 & 1.0 & $3.7688 \times 10^{-34}$ & $1.3438 \times 10^{-6}$ & & \\
\hline & 2 & 1.0 & $8.1145 \times 10^{-278}$ & $3.2898 \times 10^{-47}$ & 0.524 & \\
\hline & 3 & 1.0 & $3.7475 \times 10^{-2227}$ & $4.2454 \times 10^{-372}$ & 3.093 & 7.99 \\
\hline \multirow{3}{*}{ ZM3 } & 1 & 1.0 & $2.9165 \times 10^{-35}$ & $8.7723 \times 10^{-7}$ & & \\
\hline & 2 & 1.0 & $6.9307 \times 10^{-288}$ & $6.9039 \times 10^{-49}$ & 0.342 & \\
\hline & 3 & 1.0 & $7.0488 \times 10^{-2309}$ & $1.0162 \times 10^{-385}$ & 1.968 & 7.99 \\
\hline \multirow{3}{*}{ SFM1 } & 1 & 1.0 & $2.3448 \times 10^{-35}$ & $8.4590 \times 10^{-7}$ & & \\
\hline & 2 & 1.0 & $1.8939 \times 10^{-289}$ & $3.7890 \times 10^{-49}$ & 0.330 & \\
\hline & 3 & 1.0 & $3.4308 \times 10^{-2322}$ & $6.1405 \times 10^{-388}$ & 1.445 & 7.99 \\
\hline \multirow{3}{*}{ SFM2 } & 1 & 1.0 & $5.8129 \times 10^{-42}$ & $6.7045 \times 10^{-8}$ & & \\
\hline & 2 & 1.0 & $3.5820 \times 10^{-350}$ & $2.8707 \times 10^{-59}$ & 0.026 & \\
\hline & 3 & 1.0 & $7.4467 \times 10^{-2816}$ & $3.2431 \times 10^{-470}$ & 0.070 & 7.99 \\
\hline \multirow{3}{*}{ SFM3 } & 1 & 1.0 & $9.0965 \times 10^{-36}$ & $7.2240 \times 10^{-7}$ & & \\
\hline & 2 & 1.0 & $2.4977 \times 10^{-293}$ & $8.5486 \times 10^{-50}$ & 0.282 & \\
\hline & 3 & 1.0 & $8.0724 \times 10^{-2354}$ & $3.2870 \times 10^{-393}$ & 1.152 & 7.99 \\
\hline
\end{tabular}

TABLE 6: Convergence behavior of different iterative methods for $g_{6}(x)$.

\begin{tabular}{|c|c|c|c|c|c|c|}
\hline \multicolumn{7}{|c|}{$g_{6}(x)=\left((x-1)^{3}-1\right)^{50}, x_{0}=2.9$} \\
\hline & $\zeta$ & $x_{\zeta}$ & $\left|g\left(x_{\zeta}\right)\right|$ & $\left|x_{\zeta}-\alpha\right|$ & AEC & $\mathrm{COC}$ \\
\hline \multirow{3}{*}{ BM1 } & 1 & 2.0 & $1.5168 \times 10^{-39}$ & $5.2931 \times 10^{-2}$ & & \\
\hline & 2 & 2.0 & $1.8108 \times 10^{-381}$ & $8.0916 \times 10^{-9}$ & 0.169 & \\
\hline & 3 & 2.0 & $7.3247 \times 10^{-3096}$ & $4.1703 \times 10^{-63}$ & 6.951 & 7.93 \\
\hline \multirow{3}{*}{ AM2 } & 1 & 2.0 & $7.2090 \times 10^{-44}$ & $4.3768 \times 10^{-2}$ & & \\
\hline & 2 & 2.0 & $4.8078 \times 10^{-409}$ & $2.2725 \times 10^{-9}$ & 0.1515 & \\
\hline & 3 & 2.0 & $6.8409 \times 10^{-3308}$ & $2.3965 \times 10^{-67}$ & 168.72 & 7.93 \\
\hline \multirow{3}{*}{ ZM3 } & 1 & 2.0 & $3.1076 \times 10^{-48}$ & $3.6070 \times 10^{-2}$ & & \\
\hline & 2 & 2.0 & $1.6232 \times 10^{-450}$ & $3.3657 \times 10^{-10}$ & 0.1162 & \\
\hline & 3 & 2.0 & $4.3287 \times 10^{-3650}$ & $3.4324 \times 10^{-74}$ & 117.44 & 7.95 \\
\hline \multirow{3}{*}{ SFM1 } & 1 & 2.0 & $7.4516 \times 10^{-45}$ & $4.1903 \times 10^{-2}$ & & \\
\hline & 2 & 2.0 & $2.8683 \times 10^{-430}$ & $8.5512 \times 10^{-10}$ & 0.1425 & \\
\hline & 3 & 2.0 & $2.8294 \times 10^{-3495}$ & $4.2846 \times 10^{-71}$ & 89.952 & 7.95 \\
\hline \multirow{3}{*}{ SFM2 } & 1 & 2.0 & $9.7376 \times 10^{-71}$ & $1.3091 \times 10^{-2}$ & & \\
\hline & 2 & 2.0 & $1.8913 \times 10^{-699}$ & $3.5352 \times 10^{-15}$ & 0.0341 & \\
\hline & 3 & 2.0 & $9.3674 \times 10^{-5725}$ & $1.1023 \times 10^{-115}$ & 4.0984 & 7.99 \\
\hline \multirow{3}{*}{ SFM3 } & 1 & 2.0 & $7.2719 \times 10^{-46}$ & $4.0070 \times 10^{-2}$ & & \\
\hline & 2 & 2.0 & $2.9188 \times 10^{-442}$ & $4.9224 \times 10^{-10}$ & 0.1340 & \\
\hline & 3 & 2.0 & $1.2411 \times 10^{-3596}$ & $4.0249 \times 10^{-73}$ & 74.059 & 7.95 \\
\hline
\end{tabular}

In Table 5, we observe that our new iterative schemes SFM1, SFM2, and SFM3 perform better than the existing schemes BM1, AM2, and ZM3 for test function $g_{5}(x)$.

Example 6. In addition, we select the below given standard nonlinear test function:

$$
g_{6}(x)=\left((x-1)^{3}-1\right)^{50} \text {. }
$$

This expression has a multiple zero at $\alpha=2$ of multiplicity $k=50$. The computational results with the initial approximation $x_{0}=2.9$ are mentioned in Table 6 .
From Table 6, it is observed that computational efficiency of newly developed methods SFM1, SFM2, and SFM3is better than the earlier known methods BM1 and AM2, whereas our scheme SFM2 is more efficient as compared to ZM3. However, performance of ZM3 is comparable with the new method SFM1 for function $g_{6}(x)$.

Example 7. We use the following standard test problem involving logarithmic function: 
TABLE 7: Convergence behavior of different iterative methods for $g_{7}(x)$.

\begin{tabular}{|c|c|c|c|c|c|c|}
\hline \multicolumn{7}{|c|}{$g_{7}(x)=x^{2}\left(x^{3}-\log \left(x^{2}+1\right)\right)^{2}, x_{0}=-0.9$} \\
\hline & $\zeta$ & $x_{\zeta}$ & $\left|g\left(x_{\zeta}\right)\right|$ & $\left|x_{\zeta}-\alpha\right|$ & AEC & $\mathrm{COC}$ \\
\hline \multirow{3}{*}{ BM1 } & 1 & -0.0 & $2.1027 \times 10^{-23}$ & $1.6612 \times 10^{-4}$ & & \\
\hline & 2 & -0.0 & $7.4964 \times 10^{-184}$ & $3.0139 \times 10^{-31}$ & $3.4 \times 10^{-4}$ & \\
\hline & 3 & -0.0 & $1.9858 \times 10^{-1467}$ & $3.5453 \times 10^{-245}$ & $8.6 \times 10^{-5}$ & 7.99 \\
\hline \multirow{3}{*}{ AM2 } & 1 & -0.0 & $7.1551 \times 10^{-24}$ & $1.3880 \times 10^{-4}$ & & \\
\hline & 2 & -0.0 & $9.7548 \times 10^{-188}$ & $6.7847 \times 10^{-32}$ & $3.2 \times 10^{-4}$ & \\
\hline & 3 & -0.0 & $1.1811 \times 10^{-1498}$ & $2.2150 \times 10^{-250}$ & 0.4922 & 7.99 \\
\hline \multirow{3}{*}{ ZM3 } & 1 & -0.0 & $2.8275 \times 10^{-24}$ & $1.1890 \times 10^{-4}$ & & \\
\hline & 2 & -0.0 & $8.9533 \times 10^{-192}$ & $1.4410 \times 10^{-32}$ & $2.7 \times 10^{-4}$ & \\
\hline & 3 & -0.0 & $9.1591 \times 10^{-1532}$ & $6.7139 \times 10^{-256}$ & 0.3605 & 7.99 \\
\hline \multirow{3}{*}{ SFM1 } & 1 & -0.0 & $2.9473 \times 10^{-24}$ & $1.1973 \times 10^{-4}$ & & \\
\hline & 2 & -0.0 & $3.5576 \times 10^{-192}$ & $1.2355 \times 10^{-32}$ & $2.7 \times 10^{-4}$ & \\
\hline & 3 & -0.0 & $1.6211 \times 10^{-1535}$ & $1.5908 \times 10^{-256}$ & 0.2924 & 7.99 \\
\hline \multirow{3}{*}{ SFM2 } & 1 & -0.0 & $3.7297 \times 10^{-26}$ & $5.7801 \times 10^{-5}$ & & \\
\hline & 2 & -0.0 & $1.7193 \times 10^{-211}$ & $7.4569 \times 10^{-36}$ & $1.3 \times 10^{-4}$ & \\
\hline & 3 & -0.0 & $3.5209 \times 10^{-1694}$ & $5.7250 \times 10^{-283}$ & $5.9 \times 10^{-2}$ & 7.99 \\
\hline \multirow{3}{*}{ SFM3 } & 1 & -0.0 & $2.2186 \times 10^{-24}$ & $1.1419 \times 10^{-4}$ & & \\
\hline & 2 & -0.0 & $1.6657 \times 10^{-193}$ & $7.4176 \times 10^{-33}$ & $2.6 \times 10^{-4}$ & \\
\hline & 3 & -0.0 & $1.6993 \times 10^{-1546}$ & $2.3534 \times 10^{-258}$ & 0.2564 & 7.99 \\
\hline
\end{tabular}

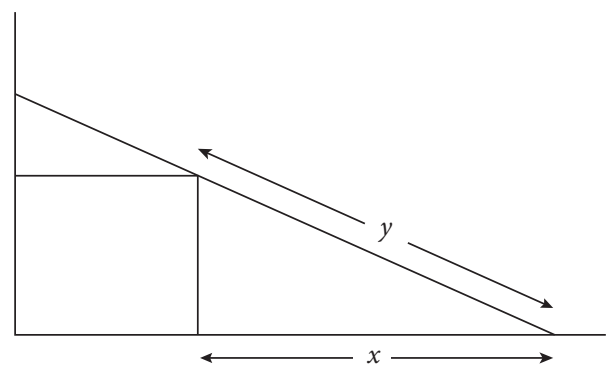

Figure 1: Beam positioning problem.



(a)

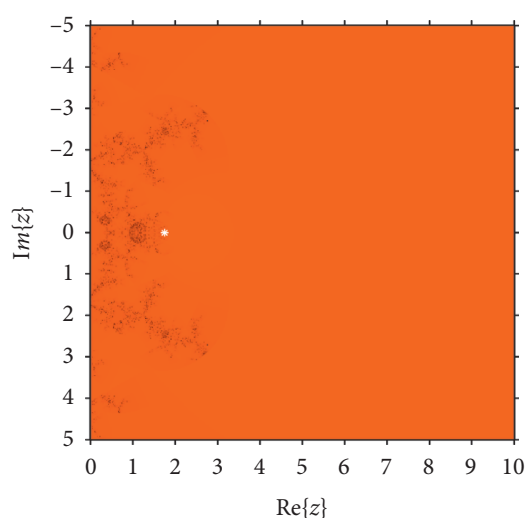

(b)

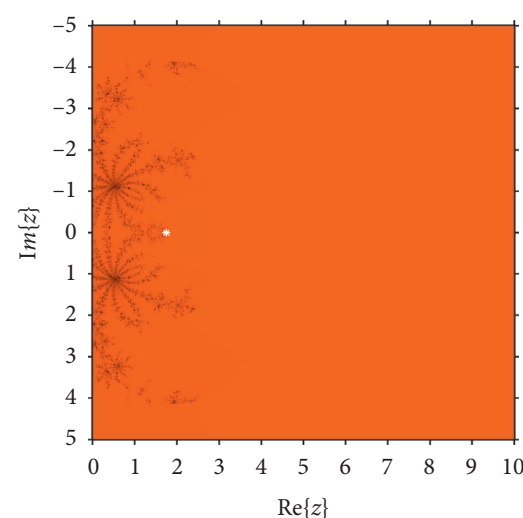

(c)

Figure 2: Basins of attraction of (a) SFM1, (b) SFM2, and (c) SFM3 for $g_{1}(x)$. 


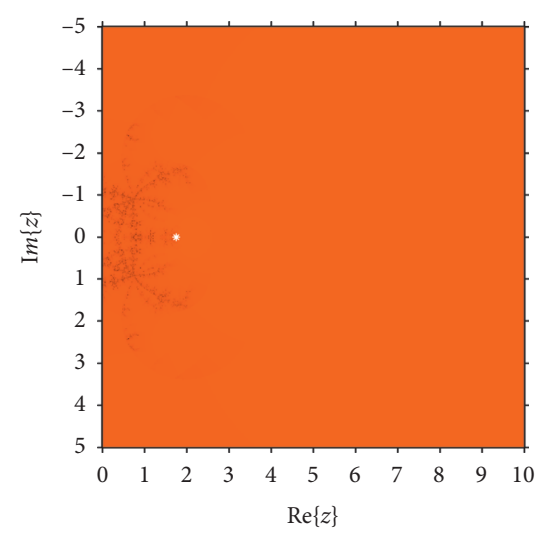

(a)

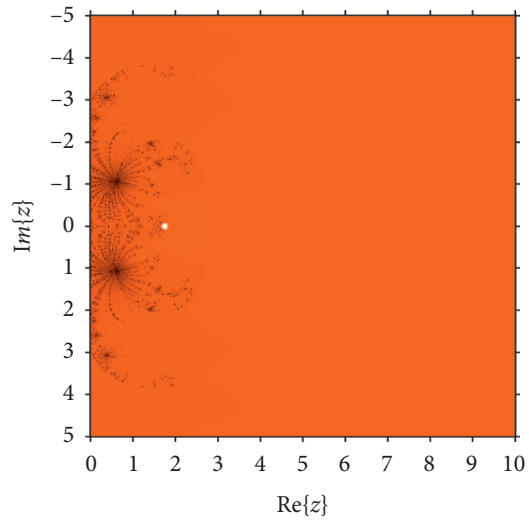

(b)



(c)

Figure 3: Basins of attraction of (a) BM1, (b) AM2, and (c) ZM3 for $g_{1}(x)$.

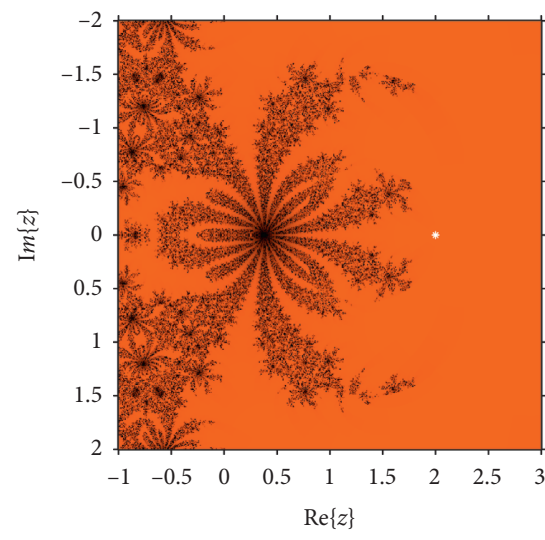

(a)



(b)

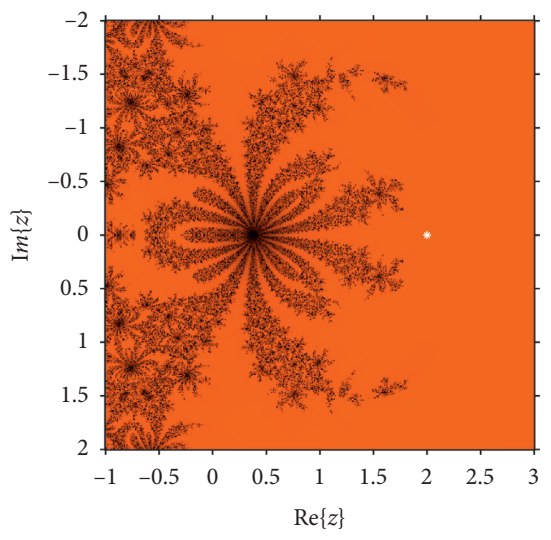

(c)

FIgURE 4: Basins of attraction of (a) SFM1, (b) SFM2, and (c) SFM3 for $g_{2}(x)$.



(a)

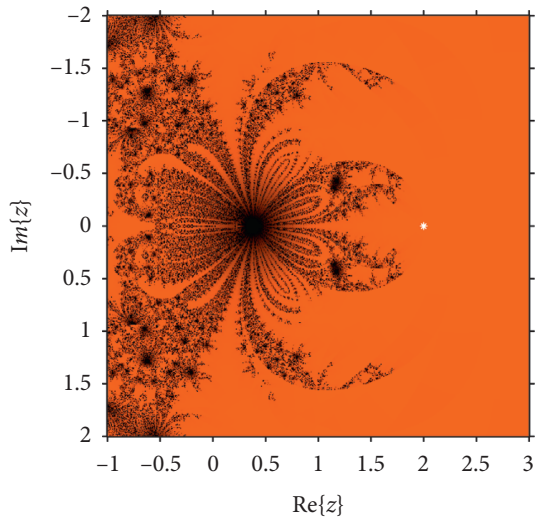

(b)



(c)

Figure 5: Basins of attraction of (a) BM1, (b) AM2, and (c) ZM3 for $g_{2}(x)$. 


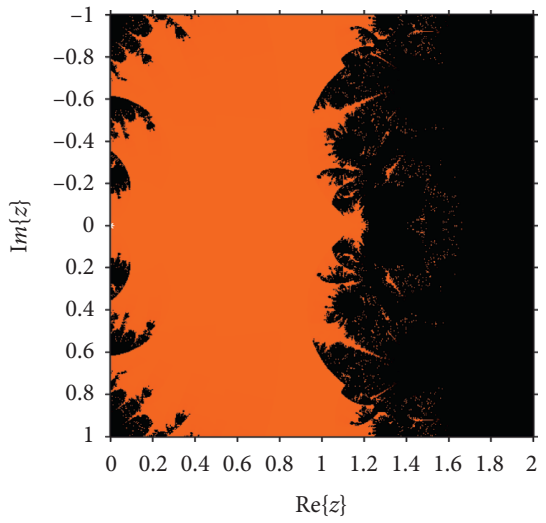

(a)



(b)

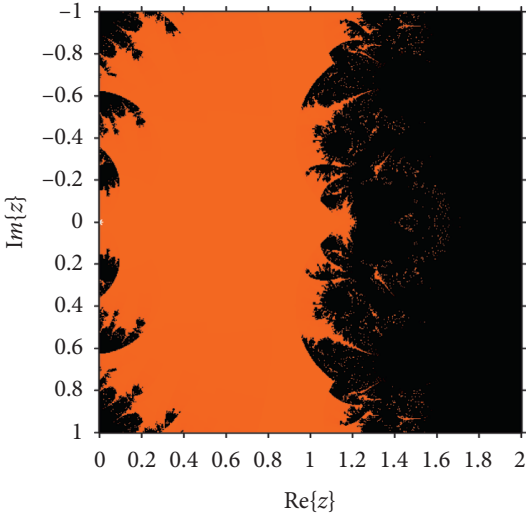

(c)

Figure 6: Basins of attraction of (a) SFM1, (b) SFM2, and (c) SFM3 for $g_{3}(x)$.

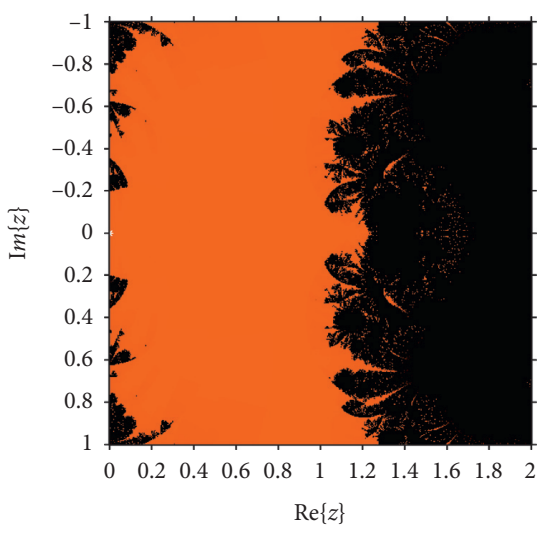

(a)



(b)

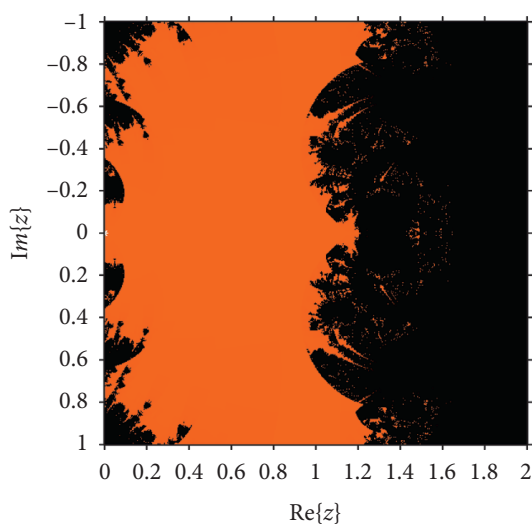

(c)

Figure 7: Basins of attraction of (a) BM1, (b) AM2, and (c) ZM3 for $g_{3}(x)$.

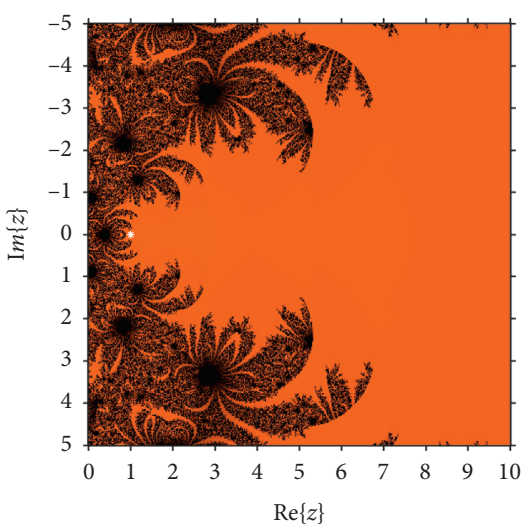

(a)



(b)

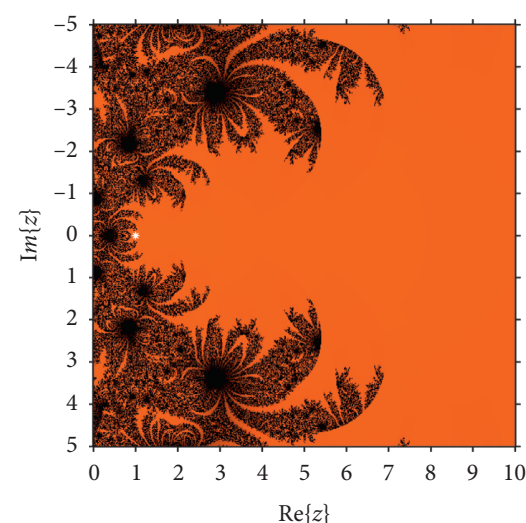

(c)

FIgURE 8: Basins of attraction of (a) SFM1, (b) SFM2, and (c) SFM3 for $g_{4}(x)$. 


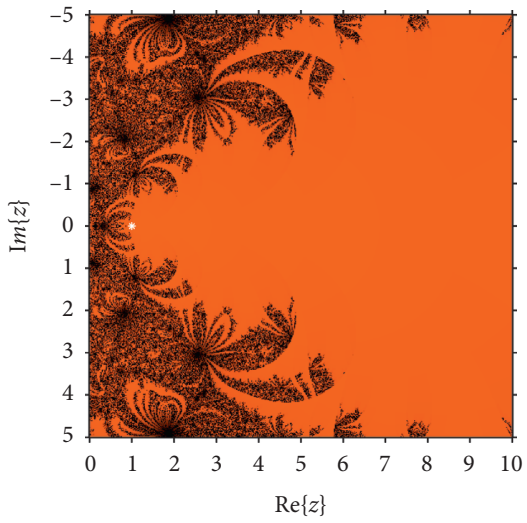

(a)

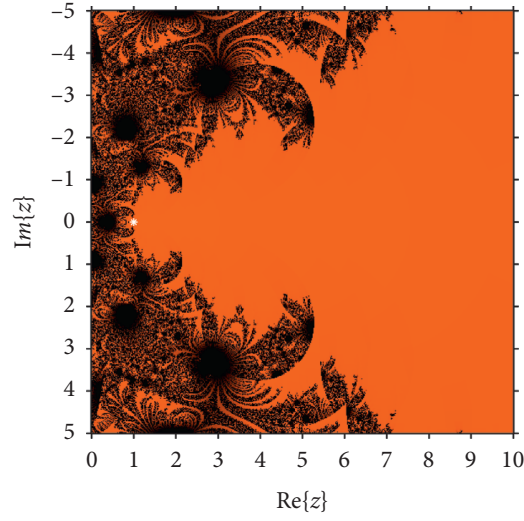

(b)

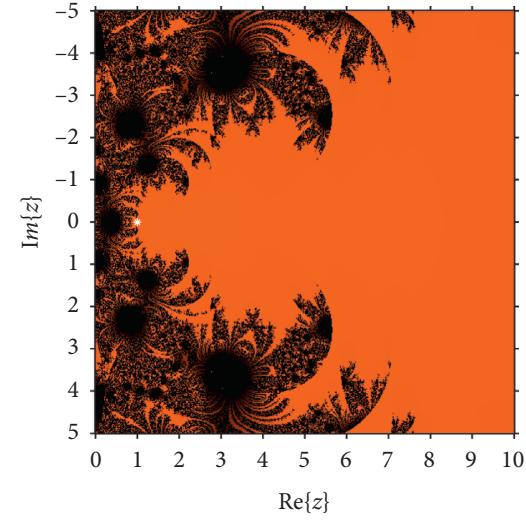

(c)

Figure 9: Basins of attraction of (a) BM1, (b) AM2, and (c) ZM3 for $g_{4}(x)$.

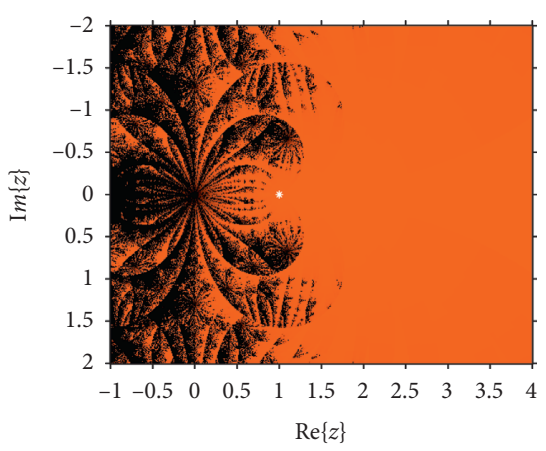

(a)

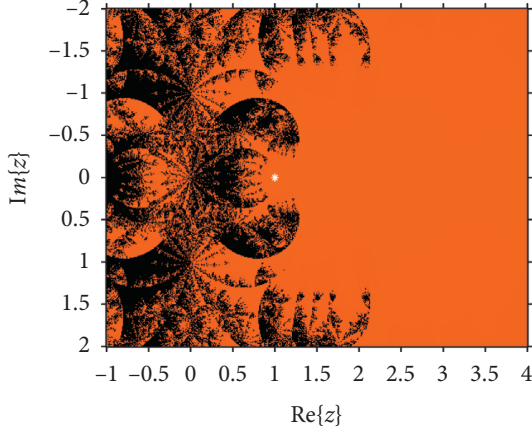

(b)

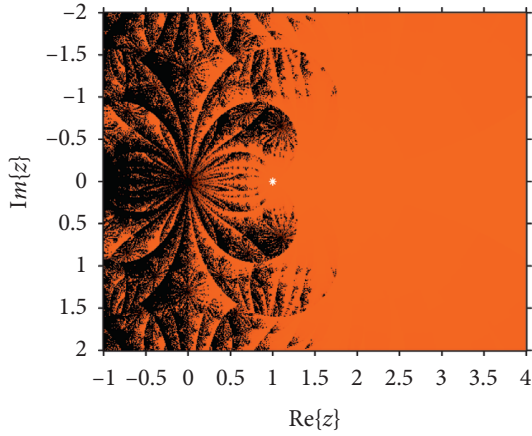

(c)

Figure 10: Basins of attraction of (a) SFM1, (b) SFM2, and (c) SFM3 for $g_{5}(x)$.



(a)

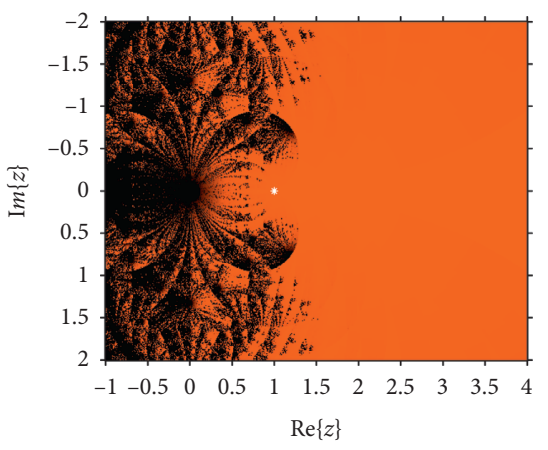

(b)

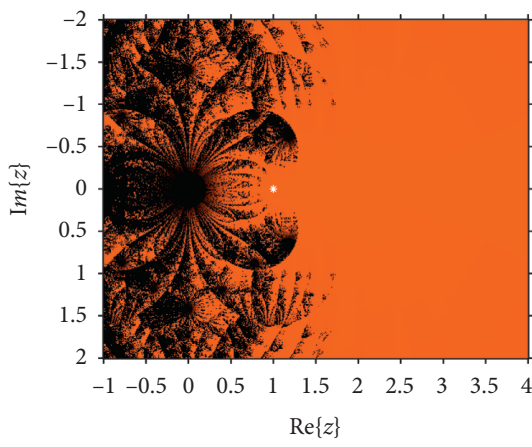

(c)

Figure 11: Basins of attraction of (a) BM1, (b) AM2, and (c) ZM3 for $g_{5}(x)$.

$$
g_{7}(x)=x^{2}\left(x^{3}-\log \left(x^{2}+1\right)\right)^{2},
$$

having multiple zero $\alpha=0.0$ of multiplicity 6 . We choose the initial guess $x_{0}=-0.9$ for the comparison results shown in Table 7.

From Table 7, it can be observed that the newly developed schemes SFM1, SFM2, and SFM3 give the faster convergence and smaller errors than the earlier known methods for test function $g_{7}(x)$ and these numerical results confirm the robust nature of our presented iterative method.

It can be seen from the results depicted in Tables 1-7 that the special cases of our newly developed methods SFM1, SFM2, and SFM3 are faster, efficient, and reliable than the existing known methods BM1, AM2, and ZM3 in terms of absolute error and computational order of convergence, etc. 




(a)

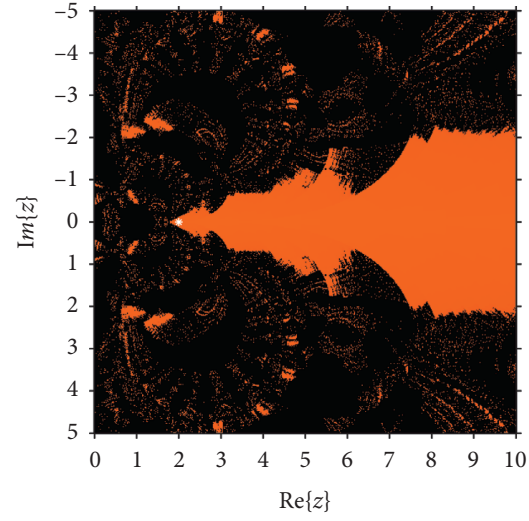

(b)

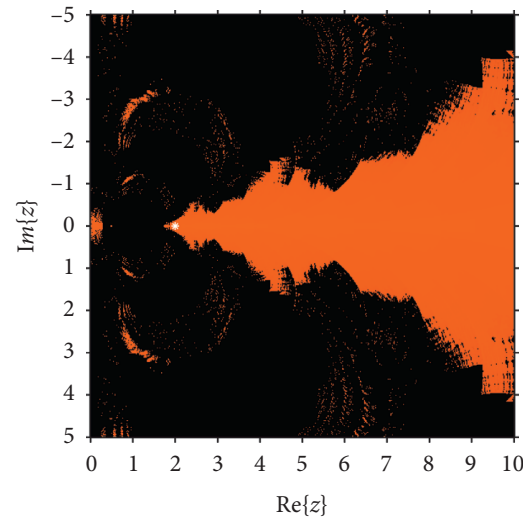

(c)

Figure 12: Basins of attraction of (a) SFM1, (b) SFM2, and (c) SFM3 for $g_{6}(x)$.

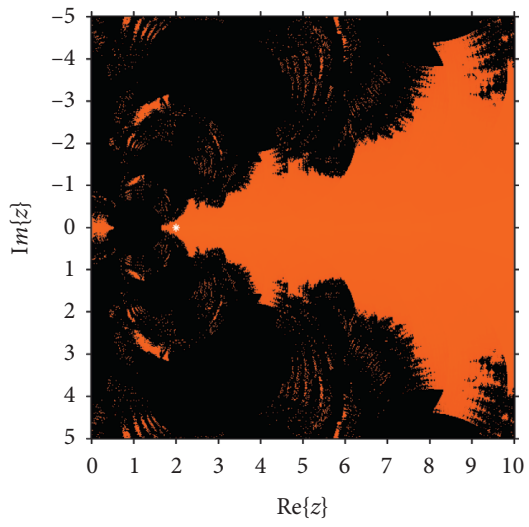

(a)

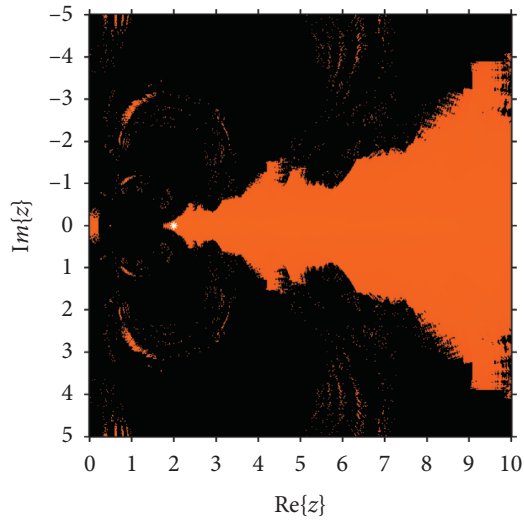

(b)

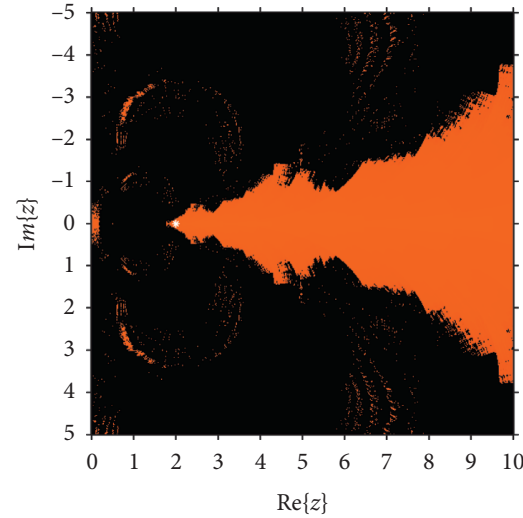

(c)

FIgURE 13: Basins of attraction of (a) BM1, (b) AM2, and (c) ZM3 for $g_{6}(x)$.

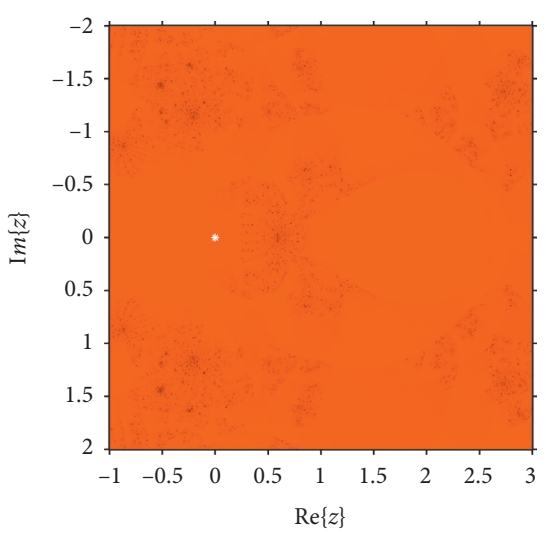

(a)



(b)



(c)

Figure 14: Basins of attraction of (a) SFM1, (b) SFM2, and (c) SFM3 for $g_{7}(x)$.

We conclude that the newly developed family gives the faster convergence, small absolute, and residual error corresponding to the involved function. It is also observed that, in most cases, SFM2 is faster among all schemes. Therefore, the new presented schemes are better alternatives to the present schemes. 


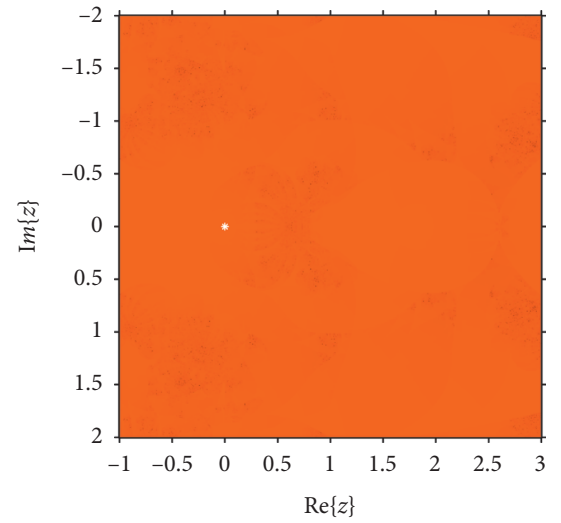

(a)



(b)

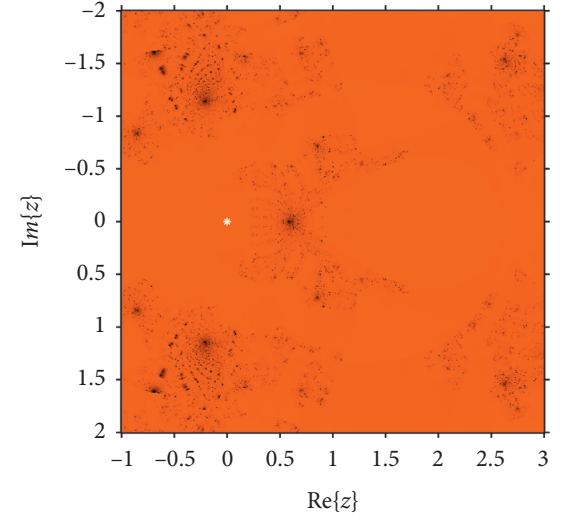

(c)

Figure 15: Basins of attraction of (a) BM1, (b) AM2, and (c) ZM3 for $g_{7}(x)$.

\section{Complex Dynamics}

Regarding the dynamical behavior, our goal is to observe and compare the performance of proposed methods by means of a graphical tool, named as the basins of attraction. Initially, Vrscay and Gilbert [23] suggest this idea to investigate the convergence and dynamical behavior of iterative schemes. For the sake of stability comparison, we plot the dynamical planes corresponding to each scheme, SFM1, SFM2, SFM3, BM1, $\mathrm{AM} 2$, and ZM3, for the nonlinear functions $g_{1}(x), g_{2}(x)$, $g_{3}(x), g_{4}(x), g_{5}(x), g_{6}(x)$, and $g_{7}(x)$ used for numerical comparison in Section 4. The complex dynamical planes presented in Figures $2-15$ have been generating by using the procedure defined in [1]. Regarding the stability comparison, a mesh of $[500 \times 500]$ points is defined in the region of the complex plane to check how wide the set of initial guess leads us to the desired repeated root. We used to work out up to a total number of 80 iterations with the stopping criterion $10^{-3}$. If the iteration sequence converges to the multiple zero, then the convergence region is painted in orange colour. The divergence region is painted in black colour, when the iteration sequence does not converge to the multiple root or converge to strange fixed points (which are not roots of nonlinear function). A white star represents the multiple zero in Figures 2-15.

Figures 2-15 show the study of the convergence and divergence regions of the new schemes SFM1, SFM2, and SFM3 in comparison with the other schemes of the same order. It is observed that our methods SFM1, SFM2, and SFM3 have more wider convergence region than the earlier ones BM1, AM2, and ZM3. In the case of $g_{1}(x), g_{2}(x)$, and $g_{6}(x)$, we observed that the new schemes SFM1, SFM2, and SFM3 are more stable than BM1, AM2, and ZM3 as they are almost divergence-free and converge faster than BM1, AM2, and ZM3 in their common regions of convergence. So, the stable and consistent nature of newly developed scheme is clear from the graphics.

\section{Conclusion}

In this paper, we have presented a new general family of optimal iterative methods for finding multiple roots of nonlinear equations with known multiplicity using weight functions. An extensive convergence analysis is presented to verify the optimal convergence of the new family. Some special cases of the family are also presented which require only three-function and one-derivative evaluations at each iteration to reach optimal eighth order convergence. A variety of numerical test functions along with some realworld problems such as beam positioning model and Van der Waals' equation of state are used to ensure that the newly developed family efficiently competes with the other existing methods. The dynamical analysis of the proposed methods is also presented to validate the theoretical results by using graphical tool, termed as the basins of attraction. As the proposed methods are sensitive towards initial guess, so for the safe selection of initial guess, convergence regions are presented. Figures 2-15 show that our newly developed schemes have wider regions of convergence. Hence, we have more choices for initial guess to choose from. Finally, our newly presented scheme shows stable behavior in complex plane and validates the theoretical results. By considering the above stated facts, it is concluded that the new methods SFM1, SFM2, and SFM3 are efficient, stable, and robust among the existing schemes of their domain.

\section{Data Availability}

No data were used to support this study.

\section{Conflicts of Interest}

The authors declare that they have no conflicts of interest.

\section{References}

[1] N. A. Mir, M. Shams, N. Rafiq, S. Akram, and R. Ahmed, "On family of simultaneous method for finding distinct as well as multiple roots of Non-Linear polynomial equation," PUJM, vol. 52, no. 6, pp. 31-44, 2020.

[2] M. S. Petkovi'c, B. Neta, L. D. Petkovic, and J. D. zunic, Multipoint Methods for Solving Non-Linear Equations, Academic Press, New York, NY, USA, pp. 635-660, 2014. 
[3] N. Rafiq, S. Akram, N. A. Mir, and M. Shams, "Study of dynamical behavior and stability of iterative methods for nonlinear equation with applications in engineering," Mathematical Problems in Engineering, vol. 2020, Article ID 3524324, 2020.

[4] M. Shams, N. A Mir, N. Rafiq, A. O. Almatroud, and S. Akram, "On dynamics of iterative techniques for nonlinear equation with applications in engineering," Mathematical Problems in Engineering, vol. 2020, Article ID 5853296, 2020.

[5] J. F. Traub, Iterative Methods for the Solution of Equations, Prentice-Hall, Englewood Cliffs, NJ, USA, 1964.

[6] L. B. Rall, "Convergence of the Newton process to multiple solutions," Numerische Mathematik, vol. 9, no. 1, pp. 23-37, 1966.

[7] R. Behl, A. Cordero, S. S. Motsa, and J. R. Torregrosa, "On developing fourth-order optimal families of methods for multiple roots and their dynamics," Applied Mathematics and Computation, vol. 265, no. 15, pp. 520-532, 2015.

[8] R. Behl, A. Cordero, S. S. Motsa, J. R. Torregrosa, and V. Kanwar, "An optimal fourth-order family of methods for multiple roots and its dynamics," Numerical Algorithms, vol. 71, no. 4, pp. 775-796, 2016.

[9] F. Soleymani, D. K. R. Babajee, and T. Lotfi, "On a numerical technique for finding multiple zeros and its dynamic," Journal of the Egyptian Mathematical Society, vol. 21, no. 3, pp. 346-353, 2013.

[10] R. Thukral, "A new family of fourth-order iterative methods for solving non-linear equations with multiple roots," Journal of Numerical Mathematics and Stochastics, vol. 6, no. 1, pp. 37-44, 2014.

[11] X. Zhou, X. Chen, and Y. Song, "Constructing higher-order methods for obtaining the multiple roots of nonlinear equations," Journal of Computational and Applied Mathematics, vol. 235, no. 14, pp. 4199-4206, 2011.

[12] R. Thukral, "Introduction to higher-order iterative methods for finding multiple roots of nonlinear equations," Journal of Mathematics, vol. 2013, Article ID 404635, 2013.

[13] Y. H. Geum, Y. I. Kim, and B. Neta, "A class of two-point sixth-order multiple-zero finders of modified double-Newton type and their dynamics," Applied Mathematics and Computation, vol. 270, pp. 387-400, 2015.

[14] Y. H. Geum, Y. I. Kim, and B. Neta, "A sixth-order family of three-point modified Newton-like multiple-root finders and the dynamics behind their extraneous fixed points," Applied Mathematics and Computation, vol. 283, pp. 120-140, 2016.

[15] J. R. Sharma, D. Kumar, and C. Cattani, "An efficient class of weighted-Newton multiple root solvers with seventh order convergence," Symmetry, vol. 11, no. 8, p. 1054, 2019.

[16] H. T. Kung and J. F. Traub, "Optimal order of one-point and multipoint iteration," Journal of the ACM, vol. 21, no. 4, pp. 643-651, 1974.

[17] A. M. Ostrowski, Solution of Equations and Systems of Equations, Academic Press, Cambridge, MA, USA, 1960.

[18] R. Behl, A. Cordero, S. S. Motsa, and J. R. Torregrosa, "An eighth-order family of optimal multiple root finders and its dynamics," Numerical Algorithms, vol. 77, no. 4, pp. 1249-1272, 2018.

[19] S. Akram, F. Zafar, and N. Yasmin, "An optimal eighth-order family of iterative methods for multiple roots," Mathematics, vol. 7, no. 8, p. 672, 2019.

[20] F. Zafar, A. Cordero, R. Quratulain, and J. R. Torregrosa, "Optimal iterative methods for finding multiple roots of nonlinear equations using free parameters," Journal of Mathematical Chemistry, vol. 56, no. 7, pp. 1884-1901, 2017.
[21] L. O. Jay, “A note on Q-order of convergence," Bit Numerical Mathematics, vol. 41, no. 2, pp. 422-429, 2001.

[22] J. L. Zachary, Introduction to Scientific Programing, Computational Problem Solving Using Maple and C, Springer, New York, NY, USA, 2012.

[23] E. R. Vrscay and W. J. Gilbert, "Extraneous fixed points, basin boundaries and chaotic dynamics for Schr der and $\mathrm{K}$ nig rational iteration functions," Numerische Mathematik, vol. 52, no. 1, pp. 1-16, 1987. 